\title{
Detecting underabundant neutralinos
}

\section{Badziak, ${ }^{a}$ A. Delgado, ${ }^{b, c}$ M. Olechowski, ${ }^{a}$ S. Pokorski ${ }^{a}$ and K. Sakurai ${ }^{d}$}

${ }^{a}$ Institute of Theoretical Physics, Faculty of Physics, University of Warsaw, ul. Pasteura 5, PL-02-093 Warsaw, Poland

${ }^{b}$ Department of Physics, 225 Nieuwland Science Hall, University of Notre Dame, Notre Dame, IN 46556, U.S.A.

${ }^{c}$ Theory Division, Physics Department CERN, CH-1211 Geneva 23, Switzerland

${ }^{d}$ Department of Physics, King's College London, London WC2R 2LS, U.K.

E-mail: Marcin.Badziak@fuw.edu.pl, antonio.delgado@nd.edu, Marek.0lechowski@fuw.edu.pl, Stefan.Pokorski@fuw.edu.pl, kazuki.sakurai@kcl.ac.uk

ABSTRACT: The electroweak sector may play a crucial role in discovering supersymmetry. We systematically investigate the patterns of the MSSM-like electroweakinos, when the neutralino relic abundance $\Omega_{\chi} h^{2} \leq 0.12$, that is, also admitting for multi-component Dark Matter, in a broad range of the parameter space. We find that for a very large range of parameters the Direct Detection experiments are/will be sensitive to underabundant neutralinos, in spite of the strong rescaling of the flux factor. The second general conclusion is that the bound $\Omega_{\chi} h^{2} \leq 0.12$ together with the LUX (XENON1T) limits for the neutralino spin independent scattering cross sections constrain the electroweakino spectrum so that the mass differences between the NLSP and the LSP are smaller than 40 (10) GeV, respectively, with important implications for the collider searches. The future Direct Detection experiments and the high luminosity LHC run will probe almost the entire range of the LSP and NLSP mass spectrum that is consistent with the bound $\Omega_{\chi} h^{2} \leq 0.12$.

KeYwords: Supersymmetry Phenomenology

ArXiv EPrint: 1506.07177 


\section{Contents}

1 Introduction 1

2 Bino-higgsino LSP $\quad 4$

$\begin{array}{lll}2.1 \text { Collider } & 11\end{array}$

3 Wino-higgsino LSP

$\begin{array}{lll}3.1 \text { Collider } & 15\end{array}$

$\begin{array}{lll}4 & \text { Bino-wino } & 17\end{array}$

$\begin{array}{llr}5 & \text { Final comments and conclusions } & 19\end{array}$

A Neutralino and chargino composition and masses 22

A.1 $|\mu| \ll\left|M_{1}\right|,\left|M_{2}\right| \quad 22$

A.2 $\left|M_{1}\right|,\left|M_{2}\right| \ll|\mu|$

A.3 $\left|M_{2}\right| \ll|\mu|,\left|M_{1}\right| \quad 26$

A.4 $\left|M_{1}\right| \ll|\mu|,\left|M_{2}\right| \quad 27$

A.5 $\left|M_{i}\right| \sim|\mu|$ with $\left|M_{j}\right| \rightarrow \infty \quad 27$

\section{Introduction}

Supersymmetry remains to be an attractive extensions of the SM even if merely for being one of the few UV complete frameworks, with no quadratic sensitivity to large mass scales. It may well be that the supersymmetric electroweak sector will play the leading role in discovering supersymmetry. Indeed, experimental searches for coloured superpartners, motivated by the issue of naturalness, have so far given null results. It is conceivable that supersymmetric models remain natural even with new coloured degrees of freedom heavier than few $\mathrm{TeV}$ (e.g. because the soft masses are correlated [1], because the Higgs mass is further protected [2-6] or it is protected by colourless degrees of freedom [7, 8]) or it could be that supersymmetry plays no role in stabilizing the weak scale (like in split supersymmetry [9-11]).

However, in models with a stable neutralino and if one accepts the thermal history of the universe, the higgsino and gaugino masses are bounded from above by the thermal overabundance of the LSP. ${ }^{1}$ So far, the interest in the supersymmetric electroweak sector has been often linked to the fact that a stable neutralino can account for the observed Dark

\footnotetext{
${ }^{1}$ Light electroweakinos are additionally motivated by the supersymmetric solution to the muon $g-2$ anomaly, see e.g. refs. $[12,13]$ for a recent works on this topic. Throughout this paper we assume the stability of the lightest neutralino and the standard scenarios of thermal history of the Universe. For example we do not consider the scenario of late entropy production, discussed e.g. in [14].
} 
Matter (DM) in the universe. It is, however, conceivable that DM has a multi-component structure. There is no reason why in the "dark" sector there would be only one stable particle. It is possible that the neutralino component in the observed DM is small or even very small and the bulk of DM has a different origin. The electroweak sector is then an interesting signature of supersymmetry in its own sake and there should be investigated the prospects for its discovery for any $\Omega_{\chi} \leq \Omega_{\mathrm{DM}}$.

Partial results of such investigations do exist in the literature, particularly for collider signatures and for the pure higgsino and wino limits [15]-[38]. ${ }^{2}$ In this paper we readdress this question, with the aim of an overall, systematic analysis in a large parameter space of the electroweak sector and with the focus on the interplay of the direct detection (DD) and collider experiments. Our global analysis leads to several new general conclusions. An additional theoretical motivation for such a global analysis is the variety of possible mechanisms for mediation of supersymmetry breaking, with different patterns of gaugino masses.

There are at least two different ways to discover those underabundant neutralinos, in DD experiments and in colliders. The latter are at present excluded by LUX [40] for

$$
\frac{\sigma_{\chi}^{\mathrm{SI}}}{\sigma_{\mathrm{LUX}}^{\mathrm{SI}}} \frac{\Omega_{\chi}}{\Omega_{\mathrm{DM}}}>1,
$$

where $\sigma_{\mathrm{LUX}}^{\mathrm{SI}}$ is the upper bound on the cross section obtained with the local DM density $\rho_{0}=0.3 \mathrm{GeV} / \mathrm{cm}^{2}$, corresponding to the observed dark matter abundance $\Omega_{\mathrm{DM}}$ [41]. For the future discovery potential in the direct detection, the $\sigma_{\mathrm{LUX}}^{\mathrm{SI}}$ has to be replaced by the corresponding new experimental limits. For the colliders one needs to know the spectrum of the electroweakinos which will then dictate the different signatures. Indirect DM detection experiments may also be sensitive to our underabundant neutralino scenario, although, as we will see later, the current limit is weaker than the direct detection limit most of the cases.

Motivated by the absence, so far, of any signal of the scalar superpartners and heavy Higgs bosons at the LHC, we will assume that they are sufficiently decoupled to be neglected in the neutralino annihilation and scattering processes. Most of the results presented here are for heavy degenerate slepton and squark masses, as for instance in the split supersymmetry models. There, the gluinos are light and the renormalization effects by strong interactions are weak. However, we have checked that our conclusions remain unchanged even when sleptons are just about $20 \%$ heavier than the LSP and we show some results for that case, too. The relevant supersymmetric parameter space consists then of the soft bino $\left(M_{1}\right)$ and wino $\left(M_{2}\right)$ masses, the higgsino mass $(\mu)$ and the ratio of the vacuum expectation values of the two Higss fields $\frac{\left\langle H_{u}\right\rangle}{\left\langle H_{d}\right\rangle}(\tan \beta)$. In our scan the above mass parameters are bounded from below by $100 \mathrm{GeV}$, so we do not consider the Higgs and $Z$ funnel effects. We also do not consider the funnel effects of the heavy CP-even $(H)$ and odd $(A)$ Higgses, because we assume they are as heavy as sfermions. Our analysis applies to the MSSM and to all models where the admixture of additional states to the LSP and NLSP mass

\footnotetext{
${ }^{2}$ More general studies based on $S$-matrix unitarity and the dynamics of thermal freeze-out can be found e.g. in [39].
} 
eigenstates is small like in certain versions of the NMSSM [42] or folded SUSY [7, 8] where one expects the higgsinos to be light. In general one can view our analysis as that of a simplified model with a singlet (the bino), a pair of doublets (the higgsino) and a triplet (the wino) of $\mathrm{SU}(2)$ with couplings close to the gauge couplings.

Our approach will be to first study certain limits of the LSP composition:

- bino-higgsino with wino decoupled; this spectrum is motivated by minimal supergravity [43, 44] or gauge mediation [45].

- wino-higgsino with bino decoupled; this spectrum may appear in theories with anomaly mediation and light higgsinos [9, 46-50].

- bino-wino with a heavy but not totally decoupled higgsino, to mediate some binowino mixing; this situation may happen in an anomaly mediated scenario with the higgs superfields acting as messengers of supersymmetry breaking [50-53].

Those limiting cases are a good reference point for subsequently investigating the general case in which higgsinos and both electroweak (EW) gauginos simultaneously contribute to the LSP composition (that might possibly occur in more sophisticated SUSY breaking schemes like e.g. mirage mediation [54, 55] or mini-split supersymmetry [51-53] ).

The main conclusions of this paper are the following: first of all, in spite of the small relic abundance and the rescaling in the flux factor, the present and future DD experiments are/will be sensitive to neutralinos with $\Omega_{\chi} h^{2}$ even two to three orders of magnitude below the observed value $\Omega_{\mathrm{DM}} h^{2}=0.12 .{ }^{3}$ Thus, a discovery of a signal in DD experiments would not necessarily mean the discovery of the main DM component but might be a strong hint for supersymmetry. Similarly, the present and future limits on the spin independent scattering cross section put strong constraints on the electroweakino parameter space with underabundant neutralinos.

Secondly, the results of the DD experiments have strong impact on the search strategies in colliders for electroweakinos in the bulk of the supersymmetric parameter space, if sfermions are heavy. This is because then the smaller the $\sigma_{\chi}^{\mathrm{SI}}$ and/or the $\Omega_{\chi}$ are/is, the smaller the mass splitting between the LSP and NLSP becomes, which is crucial for collider searches. Already the current LUX limit constrains this splitting to be less than $40 \mathrm{GeV}$. With the future direct detection experiments, such as XENON1T [56] and LZ [57], the bound on the LSP-NLSP mass splitting can be brought below $10 \mathrm{GeV}$, as a generic result for a MSSM-like spectrum with heavy sfermions. Qualitatively, that result can be understood as a consequence of a smaller bino contribution to the LSP, strongly constrained from above by the limits from the DD experiments, in addition to the constraint from the upper bound on $\Omega_{\chi} h^{2}$. Of course, if a signal was discovered in the DD experiments, similar remarks would apply to the searches for its direct confirmation in colliders.

Finally, focusing on the interplay of the DD and collider experiments, we identify the parameter regions where both may have comparable discovery potential and supplement each other and those where they are complementary to each other. There are regions of the

\footnotetext{
${ }^{3}$ This has been already pointed out for higgsino-like LSPs with $m_{\tilde{\chi}_{1}^{0}} \lesssim 350 \mathrm{GeV}[17]$.
} 
parameter space in which the LSP escapes direct detection and we survey the prospects of the $13 / 14 \mathrm{TeV}$ LHC to probe the SUSY EWkino states in these regions. In many cases existing search techniques for quasi-degenerate states at the $13 / 14 \mathrm{TeV}$ LHC are able to close the gaps in the parameter space that are inaccessible to future direct detection experiments. However, for some regions of the parameters the spin independent scattering cross section is below the neutrino background [58] and parts of those regions are also inaccessible to the $13 / 14 \mathrm{TeV}$ LHC experiments using present search techniques. We classify these regions to encourage search for new techniques to probe them at the LHC and future colliders.

\section{Bino-higgsino LSP}

The electroweakino sector with decoupled wino component in the LSP and the prospects for its experimental discovery have already been studied in a number of papers $[17,18,21-$ $23,26,28,34,35,38,59]$. In this paper we reanalyze this case for completeness of our presentation and also for stressing several general conclusions.

With heavy sfermions, pure bino has no annihilation channels and is excluded by the bound $\Omega_{\chi} h^{2} \leq 0.12$ [41]. The annihilation cross section for pure higgsino $\tilde{h}$ is determined by the vertex $\tilde{h}^{0} \tilde{h}^{ \pm} W^{\mp}$. The dominant annihilation channel is into the gauge bosons and co-annihilation with almost mass degenerate remaining higgsino states is very important. The approximate formula for the relic abundance reads [15]:

$$
\Omega_{\tilde{h}} h^{2}=0.10\left(\frac{\mu}{1 \mathrm{TeV}}\right)^{2} .
$$

Thermally produced pure higgsinos overclose the universe for $|\mu| \gtrsim 1 \mathrm{TeV}$. In the left column of figure 1 we exemplify the results of the scan over $\mu$ in the range $|\mu|=(100 \mathrm{GeV}$, $2 \mathrm{TeV})$ and $M_{1}$ in the range $(100 \mathrm{GeV}, 6 \mathrm{TeV}),{ }^{4}$ with $\left|M_{2}\right|=m_{\tilde{f}}=m_{A}=7 \mathrm{TeV},{ }^{5}$ for $\Omega_{\chi} h^{2}$ versus the neutralino mass. Throughout this paper we calculate the SUSY spectrum using SOFTSUSY [61] and compute the $\Omega_{\chi}$ and the $\sigma_{\chi}^{\text {SI }}$ using micrOMEGAs [62]. We set the values of the soft SUSY breaking parameters and the $\mu$ parameter at $7 \mathrm{TeV}$. In the plots we divide the $\left(m_{\tilde{\chi}_{1}^{0}}, \Omega_{\chi}\right)$ plane into $(45 \times 40)$ bins and keep the maximum and minimum of the scaled neutralino-nucleon cross section, $\sigma_{\chi}^{\mathrm{SI}} \cdot \Omega_{\chi} / \Omega_{\mathrm{DM}}$, if there are more than one entries in the bin. For each bin we plot two points slightly displaced indicating these maximum and minimum values with the colour-coding that represents the value of $\sigma_{\chi}^{\mathrm{SI}} \cdot \Omega_{\chi} / \Omega_{\mathrm{DM}}$. We use five different markers to classify the constraint/sensitivity on the cross section versus neutralino mass plane. The scaled cross section $\sigma_{\chi}^{\mathrm{SI}} \cdot \Omega_{\chi} / \Omega_{\mathrm{DM}}$ is: larger than the LUX limit and excluded for stars, allowed by LUX but within the reach of XENON1T for circles,

\footnotetext{
${ }^{4}$ Since only relative signs of $M_{1}, M_{2}$ and $\mu$ are important, we choose $M_{1}>0$ as our convention. The lower bound of the scan over $|\mu|$ corresponds approximately to the lower experimental limit for the chargino mass. For $M_{1}<100 \mathrm{GeV}$, the LSP is bino-dominated and the bound $\Omega_{\chi} h^{2} \leq 0.12$ can be satisfied only for the LSP mass close to the half of the $Z$ or $h$ mass, where the resonant annihilation takes place. Such scenario was studied in detail in refs. [59, 60].

${ }^{5}$ As we will see in section 5 , our result is not sensitive to the precise value of the sfermion mass $(7 \mathrm{TeV})$ in our scan. Although we do not impose the Higgs mass constraint in our sample, one can tune the stop mass to fit the lightest CP-even Higgs mass to the observed value without changing the result.
} 
beyond the reach of XENON1T but within the reach of LZ for diamonds, beyond the reach of LZ but above the neutrino background floor for triangles and below the neutrino floor for crosses. ${ }^{6}$ We use the expected sensitivity of XENON1T corresponding to a three year run from 2015 and that of LZ also corresponding to a 3-year run with a projected start date of 2017. The projected sensitivities are taken from [58].

In the scanned parameter range, there is only a small admixture of the wino component (although it is not totally decoupled). As it can be seen in those plots, the minimal value of $\Omega_{\chi} h^{2} \approx 10^{-3}$ corresponds to $|\mu|=100 \mathrm{GeV}$. For a given value of the neutralino mass, approximately equal $|\mu|$ in the whole range of $\Omega_{\chi} h^{2} \leq 0.12$, the approximately pure higgsino case, $M_{1} \gg|\mu|$, corresponds to the smallest values of $\Omega_{\chi}$, that is to the boundary of the scan. The neutralino relic abundance increases with the increasing bino component, that is for a decreasing value of $M_{1}$, eventually reaching the observed value $\Omega_{\mathrm{DM}} h^{2}=0.12$. There, $|\mu| \approx M_{1}$, which is called the well tempered bino/higgsino region [15].

It is instructive to present the results of the scan in the $\left(|\mu|, M_{1}\right)$ plane as in the right column of figure 1. First of all, we indeed see that the bound $\Omega_{\mathrm{DM}} h^{2}=0.12$ corresponds to $M_{1} \approx|\mu|$ with $M_{1}<|\mu|$ and $M_{1}>|\mu|$ for low and close to $1 \mathrm{TeV}$ values of $|\mu| \approx m_{\tilde{\chi}_{1}^{0}}$, respectively. This is easily understandable as the effect of the $\mu$ dependence of the pure higgsino relic abundance, eq. (2.1). Secondly, it is interesting to compare the contours of constant $\Omega_{\chi} h^{2}$ in the plots in the right column of figure 1 with the results in the plots in the left column, remembering that $m_{\tilde{\chi}_{1}^{0}} \approx|\mu|$. A given value of $\Omega_{\chi} h^{2}$ can be obtained only for a certain range of $m_{\tilde{\chi}_{1}^{0}} \approx|\mu|$ and this is clearly seen in both sets of plots. Furthermore, the bottom-up increase of $\Omega_{\chi} h^{2}$ with the increasing bino component corresponds to the top down change of $M_{1}$ for fixed $\mu$.

Also in figure 1 there is given information about the magnitude of the spin independent scattering cross section to the nucleons and the present exclusion regions and the regions accessible in the future, in the direct detection experiments. Since the $h \chi \chi$ vertex vanishes for pure states, the tree level $\sigma_{\chi}^{\text {SI }}$ vanishes for pure higgsino. The loop effects included in the micrOMEGAs code and the residual mixing effects give non-zero but very small cross sections along the boundary of the scan in the $\left(m_{\tilde{\chi}_{1}^{0}}, \Omega_{\chi} h^{2}\right)$ plane. For a given neutralino mass, the larger the value of $\Omega_{\chi}$ is, the larger the event rate in the spin independent scattering cross section becomes because of the flux factor $\frac{\Omega_{\chi}}{\Omega_{\mathrm{DM}}}$ but also because of the larger bino-higgsino mixing angle. One can see the difference in the magnitude of the spin independent scattering cross sections between small and large values of $\tan \beta$ and between the two signs of $\mu$, especially for small $\tan \beta$. They can be understood in terms of the formulae for the mass eigenstates and the bino-higgsino mixing angle, collected for the sake of easy reference in the appendix. Approximately, the $\sigma_{\chi}^{\mathrm{SI}}$ reads [16]:

$$
\sigma_{\chi}^{\mathrm{SI}}=8 \times 10^{-45} \mathrm{~cm}^{2}\left(\frac{c_{h \chi \chi}}{0.1}\right)^{2}
$$

\footnotetext{
${ }^{6}$ If the neutralino-nucleon scattering cross section becomes smaller than some threshold, so-called the neutrino floor, the signal is swamped by the overwhelming neutrino background and the traditional technique for the direct DM detection does not work efficiently. However, this parameter region may be searched for by more creative technique, discussed e.g. in [63].
} 

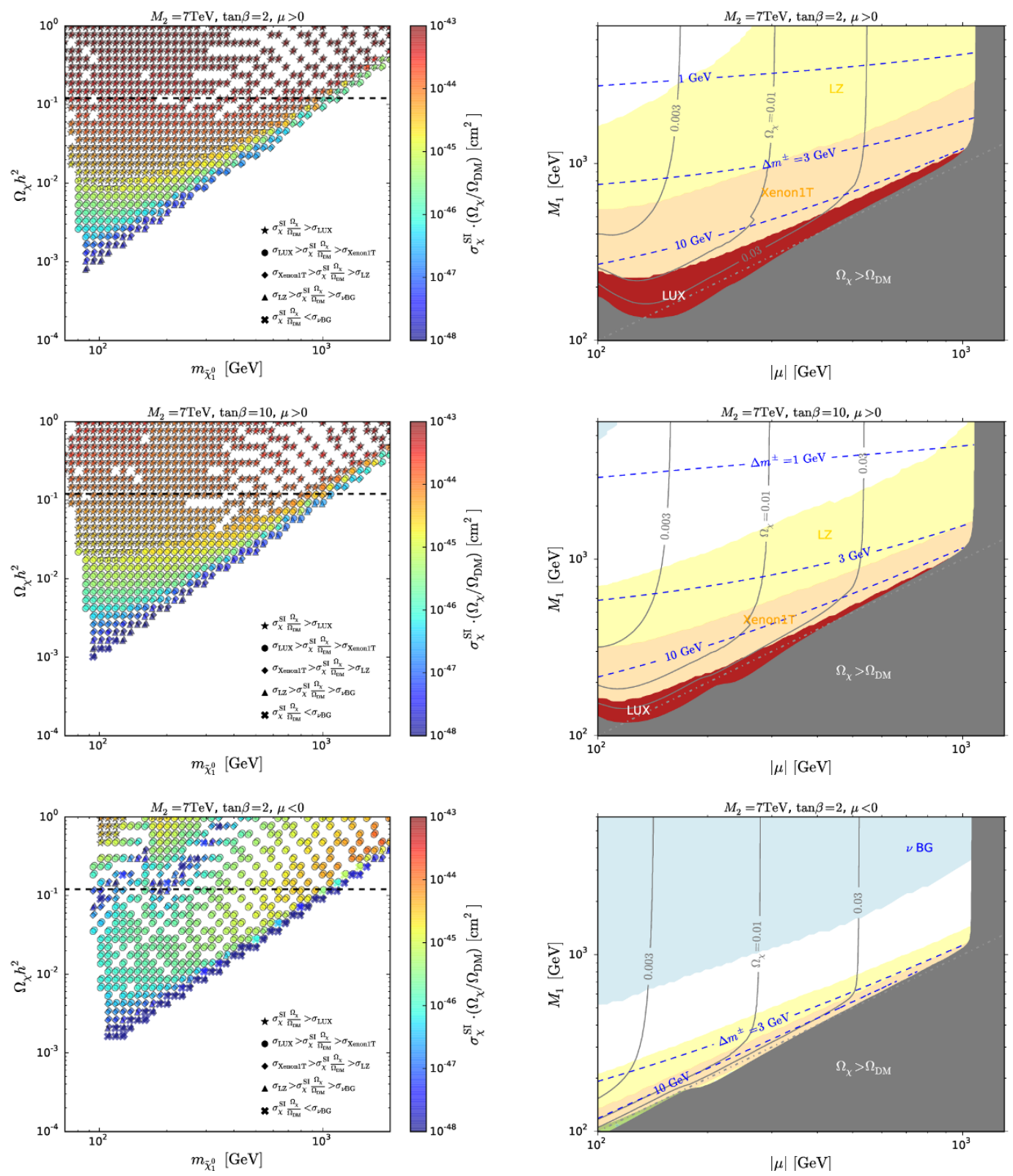

Figure 1. Some results of the scan over $M_{1}$ and $\mu$ with winos decoupled $\left(M_{2}=7 \mathrm{TeV}\right)$. In the plots on the right, the white region is not accessible in the XENON1T and LZ experiments but the cross sections are above the neutrino background, and the light blue region is below that background. The red, orange and yellow regions are: excluded by LUX, accessible by XENON1T and accessible by LZ experiments, respectively. The dashed-dotted lines mark $M_{1}=|\mu|$. All other details of the plots are explained in the figures. The small green region at $M_{1} \sim|\mu|<200 \mathrm{GeV}$ in the lower right plot is the excluded region by the ICECUBE experiment [64]. 
where the coupling $c_{h \chi \chi}$ is defined as $\mathcal{L} \sim \frac{1}{2} c_{h \chi \chi}\left(\chi \chi+\chi^{\dagger} \chi^{\dagger}\right) h$ and in the limit $M_{Z} \sin \theta_{W} \ll$ ||$M_{1}|-| \mu||$ reads:

$$
c_{h \chi \chi} \approx \frac{g_{1}}{2} \sin \theta_{W} M_{Z} \frac{M_{1}+\mu \sin (2 \beta)}{\mu^{2}-M_{1}^{2}}
$$

for $|\mu|>\left|M_{1}\right|$ (this is the region almost totally excluded by the bound $\Omega_{\chi} h^{2} \leq 0.12$ ) and

$$
c_{h \chi \chi} \approx \frac{g_{1}}{4} \sin \theta_{W} M_{Z} \frac{1+\operatorname{sgn}\left(M_{1} \mu\right) \sin (2 \beta)}{M_{1}-\operatorname{sgn}\left(M_{1} \mu\right) \mu}
$$

for $|\mu|<\left|M_{1}\right|$ (see eqs. (A.13) and (A.47) as well as the corresponding comments in the appendix). Moreover, in the limit $\left|M_{1}\right|+|\mu| \gg M_{Z} \sin \theta_{W} \gg|| M_{1}|-| \mu||$, that is along the diagonal $\left|M_{1}\right| \approx|\mu|$, one has:

$$
c_{h \chi \chi} \approx \frac{\sqrt{2} g_{1}}{4} \operatorname{sgn}\left(M_{1}\right)\left[\sqrt{1+\operatorname{sgn}\left(M_{1} \mu\right) \sin (2 \beta)}-\frac{1}{\sqrt{2}} \frac{M_{Z} \sin \theta_{W}}{\left|M_{1}\right|+|\mu|}\left(1-\operatorname{sgn}\left(M_{1} \mu\right) \sin (2 \beta)\right)\right]
$$

(see eq. (A.55) and the following discussion in the appendix).

Those formulae explain the behaviour of $\sigma_{\chi}^{\mathrm{SI}}$, its $\tan \beta$ dependence and the dependence on the sign of $\mu$ for small $\tan \beta$. An important effect is the existence of the so-called blind spots for the $h \chi \chi$ coupling, ${ }^{7}$ that is the parameter regions where it is very small, for small $\tan \beta$ and $\mu M_{1}<0$, as it is evident from the above equations [16].

The main conclusion following from figure 1 is that, in spite of the rescaling by the flux factor $\frac{\Omega_{\chi}}{\Omega_{\mathrm{DM}}}$, the whole range of $\Omega_{\chi} h^{2}$ values between $10^{-3}$ and 0.12 is reachable in the direct detection experiments. A large part of the $\left(\mu, M_{1}\right)$ plane is either already ruled out by the present DD experiments or will be tested by the XENON1T and LZ experiments or at least corresponds to the spin independent cross sections above the neutrino background. The only exception is the large blind spot region for small $\tan \beta$ and $\mu<0$, not accessible in the DD experiments. We also note that (2.5) gives a blind spot near the diagonal $\left|M_{1}\right| \approx|\mu|$ for small $\tan \beta$ and $\mu<0$.

The results shown in figure 1 also confirm the known results $[15,16]$, that the binohiggsino LSP can still account for the observed DM. For large $\tan \beta$ with positive (negative) $\mu$ that would be a well-tempered bino-higgsino in a mass range from 800 (700) up to $1000 \mathrm{GeV}$ corresponding to a pure higgsino. For small $\tan \beta$ and negative $\mu$ the whole range of masses between 100 and $1000 \mathrm{GeV}$ is still allowed. The latter follows from the fact that for small $\tan \beta$ the well-tempered neutralino is close to the blind spot for the $h \chi \chi$ coupling. For small $\tan \beta$ and positive $\mu$ the $h \chi \chi$ coupling is enhanced by the $\mu \sin (2 \beta)$ term in (2.4) so only masses very close to $1 \mathrm{TeV}$, corresponding essentially to pure higgsino LSP, are allowed.

From eq. (2.4), one can see that for $M_{1} \gg|\mu|, \sigma_{\chi}^{\mathrm{SI}} \approx \frac{1}{M_{1}^{2}}$. The exclusion/accessibility regions depend on the experimental limits which are in addition a function of the LSP mass (weaker bounds for heavier LSP). The interplay of both factors explains the pattern observed in the right column of figure 1.

\footnotetext{
${ }^{7}$ Another type of blind spots involving cancellation between the light and heavy Higgs exchanges has also been discussed e.g. in [65-67].
} 
We also check the indirect DM detection experiments in our parameter scan. The indirect detection bound is usually not as strong as that for direct detections. However, it can effectively constrain the spin-dependent scattering cross section. Since the structures of spin-dependent and spin-independent cross sections are not correlated to each other, the spin-dependent bound may become superior in the blind spot region of the spin-independent cross section.

One of the most stringent bounds on the spin-dependent cross section comes from the ICECUBE experiment. ICECUBE looks for the neutrinos coming from the annihilation of the neutralinos captured by the sun. When the capture and annihilation reach the equilibrium, the total annihilation of the neutralinos is fixed by the capture rate, which is proportional mainly to the spin-dependent cross section and the local neutralino density on the sun's trajectory. Therefore, if the neutralino annihilation produces neutrinos (e.g. $\tilde{\chi}_{1}^{0} \tilde{\chi}_{1}^{0} \rightarrow W^{+} W^{-}$), the limit on the high energy neutrino flux from the sun can be translated to the limit on the spin-dependent cross section times the local neutralino density [68]. ${ }^{8}$

We have checked the spin-dependent cross section at each parameter point of our scan and confronted it with the ICECUBE spin-dependent bound shown in figure 2 of ref. [64] (obtained assuming ${ }^{9} \tilde{\chi}_{1}^{0} \tilde{\chi}_{1}^{0} \rightarrow W^{+} W^{-}$). In ref. [64] the limits on the capture rate are converted into the limits on the spin dependent cross section assuming the standard local dark matter density of $0.3 \mathrm{GeV} / \mathrm{cm}^{3}$. For smaller DM density the bounds for spin dependent cross section given in that figure 2 of ref. [64] have to be rescaled by $\Omega_{\chi} / \Omega_{D M}$ and are, of course, weaker. Another important point for understanding our results is the dependence of the SD scattering cross section on the LSP composition. The SD cross section is dominated by the $Z$ boson exchange and the $Z \chi \chi$ coupling is proportional to $\left(N_{13}^{2}-N_{14}^{2}\right)$. This coupling vanishes for any of the pure neutralino states (there is no direct $Z$-wino-wino or $Z$-bino-bino coupling while in the case of a pure higgsino this follows from the equality $N_{13}^{2}=N_{14}^{2}=\frac{1}{2}$ ). For the higgsino-dominated LSP its coupling to $Z$, given by eq. (A.14), is non-zero and suppressed by $M_{1}$ and $M_{2}$. Thus, the smaller $M_{1}$ and/or $M_{2}$ (i.e. the larger the wino and/or bino component), the larger the $Z \chi \chi$ coupling and the SD cross section, up to the point where the higgsino component becomes very small (in this limit the $Z \chi \chi$ coupling is given by eqs. (A.30) and (A.31)). Thus, the $Z \chi \chi$ coupling becomes sizable for bino-higgsino or wino-higgsino mixed LSP (see also eq. (A.56)) but in the second case the neutralino relic abundance is smaller and after the rescaling the bounds on the SD cross section are weaker. This is why in our plots the ICECUBE bounds are relevant only for the bino-higgsino mixed LSP. We found that the ICECUBE spin-dependent constraint is less stringent than the LUX spin-independent constraint in most of the parameter region. We only found small excluded regions by ICECUBE around the $M_{1} \sim|\mu|<200 \mathrm{GeV}$ in the $\left(M_{1}, \mu\right)$ plane with $\tan \beta=2, \mu<0$. This can be understood from eq. (A.56). The leading term of the $Z \chi \chi$ coupling is suppressed by $\left|M_{1}\right|+|\mu|$ and enhanced when $M_{1} \mu<0$, especially for small values of $\tan \beta$. Those regions happen to be the ones where the blind spot cancellation in the spin-independent cross section is operative. They can be seen as the green regions in the lower right plot of figure 1 and the upper left plot of figure 3.

\footnotetext{
${ }^{8}$ We thank Marc Kamionkowski for discussion on this point.

${ }^{9}$ We have checked that this is indeed the dominant annihilation process in the majority of the parameter region of our scan.
} 


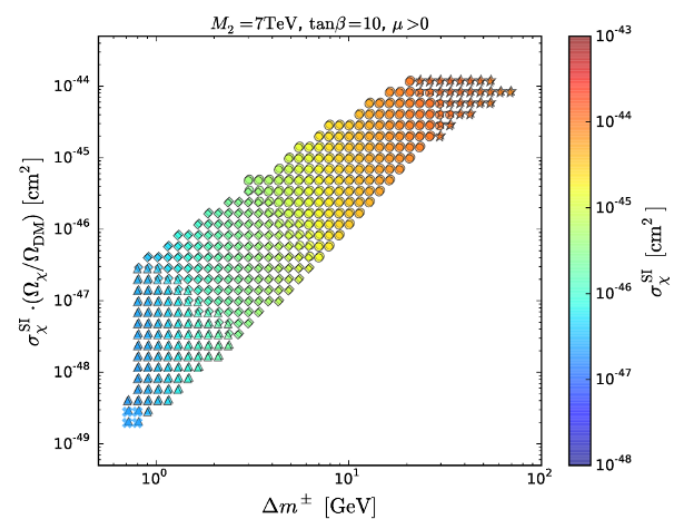

Figure 2. The spin independent scattering cross section renormalized by the proper flux factor as a function of the NLSP-LSP mass difference. The meaning of different marks is the same as in figure 1

We have checked that they are the only regions where the ICECUBE constraint is more stringent than the LUX one throughout our analysis presented in this paper.

For collider searches, an important information is the mass difference between the NLSP (usually chargino) and the LSP as well as between $\chi_{2}^{0}$ and the LSP, and also the life time of the NLSP. In figure 1 we show the contours of $\Delta m^{ \pm} \equiv m_{\tilde{\chi}_{1}^{ \pm}}-m_{\tilde{\chi}_{1}^{0}}$ in the scanned region. In figure 2 we plot the points with $\Omega_{\chi} h^{2}<0.12$ from our scans in the $\left(\Delta m^{ \pm}\right.$, $\left.\sigma_{\chi}^{\mathrm{SI}} \frac{\Omega_{\chi}}{\Omega_{\mathrm{DM}}}\right)$ plane for $\tan \beta=10$ and $\mu>0$. The colour-coding indicates $\sigma_{\chi}^{\mathrm{SI}}$ and we use the same markers as used in the left column of figure 1 to classify the DD constraint/sensitivity. One can see that after imposing the LUX bound the mass differences $\Delta m^{ \pm}$are in the range (1-30) $\mathrm{GeV}$ and the expected sensitivity of the XENON1T will bring them down to (1-10) $\mathrm{GeV}$. This is the general result for most of the parameter space, except for small $\tan \beta$ and $\mu<0$ region where the mass difference will remain to be up to $40 \mathrm{GeV}$ even after the XENON1T and LZ results.

The results can be qualitatively understood from eq. (A.5) in the appendix. We see that smaller the spin independent cross section is, smaller the mass difference between the NLSP and LSP is. For $M_{1} \gg|\mu|$, the former decreases like $\frac{1}{M_{1}^{2}}$ and the latter as $\frac{1}{M_{1}}$. We note, however, that the decoupling of the wino and bino components in the formulae for the gaugino masses and mass differences is slow, only linear in $M_{2}$ and/or $M_{1}$. Even for $M_{1}=M_{2}=7 \mathrm{TeV}$ one is still far from the pure higgsino limit. For pure higgsino states, the tree level mass difference between the lighter chargino and the LSP neutralino is zero. The loop effects give the mass difference around $350 \mathrm{MeV}[73]^{10}$ whereas in figure 1 it is at least twice that large. In the parameter range of figure 1, the chargino life time $c \tau$ is shorter than $10^{-1} \mathrm{~cm}$, where the upper bound corresponds to the smallest mass difference. The mass difference between $\tilde{\chi}_{2}^{0}$ and the LSP is about twice as large as between $\tilde{\chi}_{1}^{+}$and the LSP (see eq. (A.4)). The implications of all those facts for collider searches are discussed in the next subsection.

\footnotetext{
${ }^{10}$ For the higgsino mass around $100 \mathrm{GeV}$ the chargino-LSP mass splitting is somewhat smaller, around $250 \mathrm{MeV}$, due to corrections of order $\mathcal{O}\left(m_{Z}^{2} / m_{L S P}^{2}\right)$.
} 

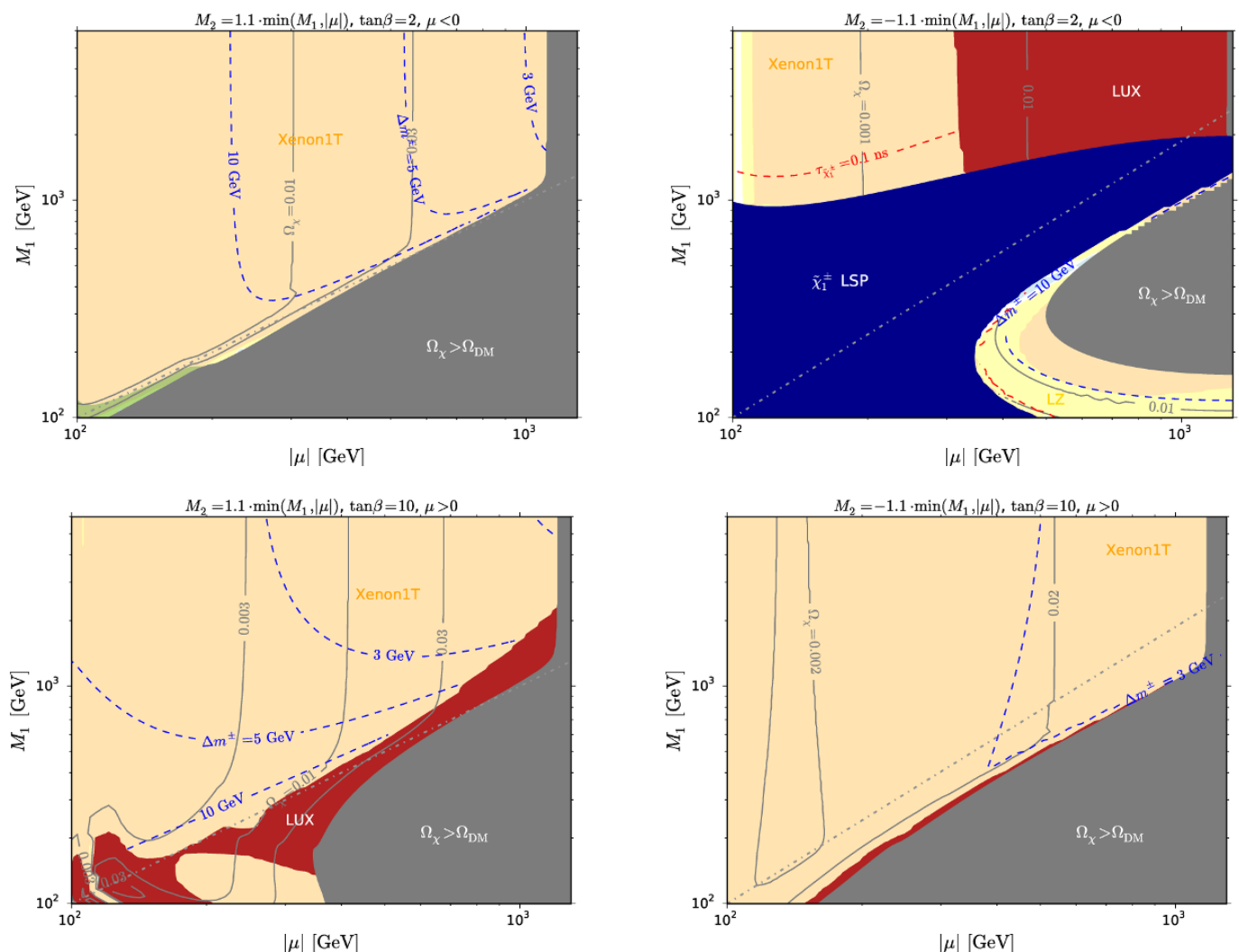

Figure 3. Same as the plots on the right of figure 1 but for non-decoupled winos. The values of $M_{2}$ are marked on the plots. The NLSP life-time is calculated following [69] with the help of SUSYHIT program [70-72]. In the dark blue region the lighter chargino is the LSP. The small green region at $M_{1} \sim|\mu|<200 \mathrm{GeV}$ in the upper left plot is the excluded region by the ICECUBE experiment [64].

With the discussed above patterns for large $\left|M_{2}\right|$ (small wino component) as a reference frame, it is interesting to consider how those patterns change when the value of $\left|M_{2}\right|$ decreases and approaches the values of $|\mu|$ and/or $M_{1}$. In figure 3 we show some generic examples of the results of the scan over $M_{1}$ and $\mu$, with $M_{2}= \pm 1.1 \min \left(M_{1},|\mu|\right)$. The plots for both signs of $M_{2}$ and $\mu$ flipped are similar to those shown in figure 3 . The blue region in the plot for $\tan \beta=2, \mu<0$ is excluded because the lighter chargino is the LSP and after the change of signs of $M_{2}$ and $\mu$ similar region is excluded by the LUX bound. The main effect is that in the $M_{1}<|\mu|$ region the $10 \%$ degeneracy $M_{2}= \pm 1.1 M_{1}$ introduces some wino component in the LSP. The large wino annihilation cross section ensures that even with a small admixture of winos, the previously excluded regions (see figure 1) are now partially allowed. This effect is weaker for opposite signs of $M_{2}$ and $\mu$ since the admixture of wino is then smaller.

Another very important effect of a larger wino component in the LSP are generically larger values of the spin independent scattering cross sections (the blind spots disappear), so that neutralinos with the relic abundance in the whole range of $\Omega_{\chi} h^{2}$ between $10^{-3}$ and 0.12 are mostly within the reach of XENON1T. Finally, the $\Delta m^{ \pm}$remains below $\mathcal{O}(10) \mathrm{GeV}$ 


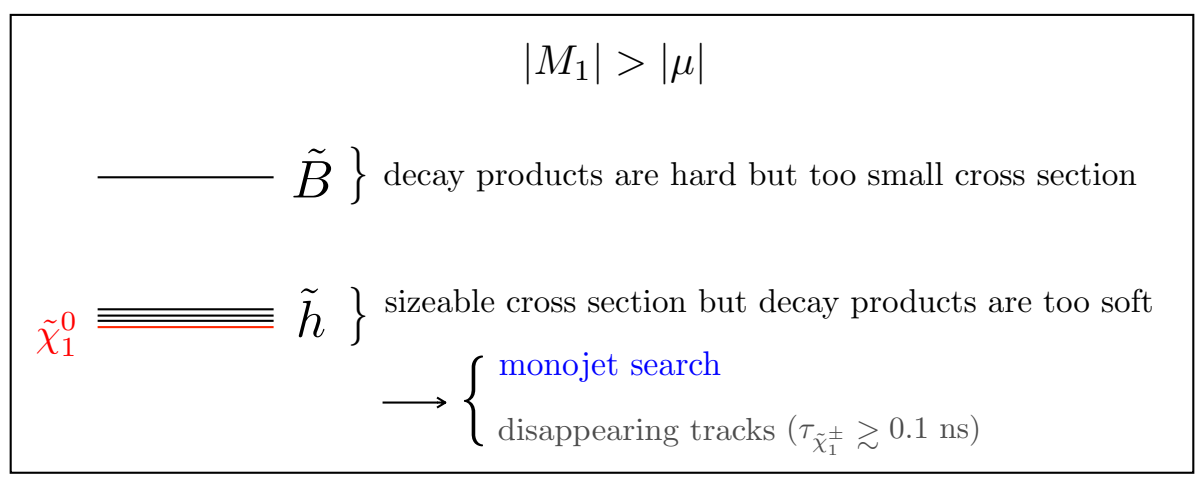

Figure 4. Strategy for collider search.
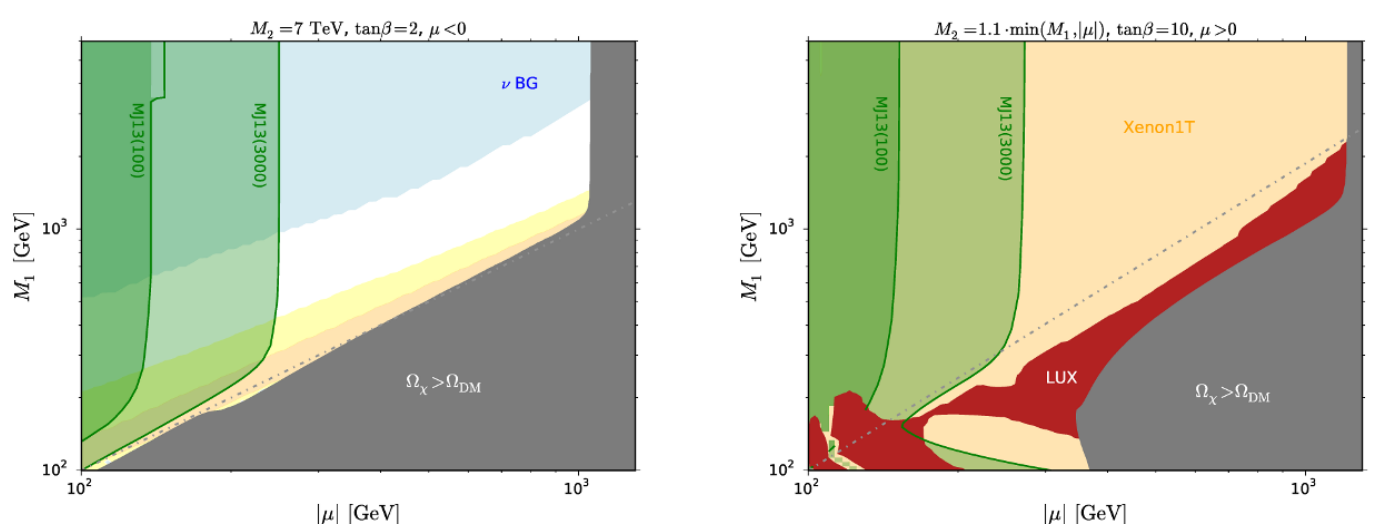

Figure 5. The estimated LHC discovery limits for decoupled winos

and $\Delta m^{0} \equiv m_{\tilde{\chi}_{2}^{0}}-m_{\tilde{\chi}_{1}^{0}} \approx 2 \Delta m^{ \pm}$(for the higgsino-dominated LSP this can be easily seen from eqs. (A.4) and (A.5) in the appendix).

\subsection{Collider}

We have shown that in the bino-higgsino scenario the region where $|\mu| \gg M_{1}$ is excluded by the overproduction of the thermal DM or the direct DM detection limit given by the LUX experiment. The conventional technique for the collider search is to focus on the production of heavier states and look for the energetic particles and missing energy originated from the decays of the produced particles. This strategy however does not work very efficiently in the phenomenologically allowed region, $M_{1}>|\mu|$ and $|\mu| \gtrsim M_{1}$. In the former case the production cross section of the heavier state namely the bino-like state is too small if squarks are decoupled. In the latter case the mass difference between the higgsino-like states and the bino-like LSP is small and the sensitivity of the conventional searches is degraded because of the softness of the decay products of the higgsino-like states, although some alternative channels based on radiative photon decays may work [29]. The same is also true for the $M_{1}>|\mu|$ case for the events with production of higgsino-like states, since higgsino-like states are almost mass degenerate and the decays among these states only produce very soft particles. 
The production of such quasi mass degenerate particles can nevertheless be detected if the mass difference between the charged state and the LSP is small enough so that the charged particle has a collider scale lifetime ( $\gtrsim 0.1 \mathrm{~ns}$ ). Such long-lived charginos may leave the distinctive disappearing track signature in the trackers. We however found that higgsino-like states are almost always short lived in our scan except for the vicinity of the chargino LSP region shown in the up-right plot of figure 3.

Another way of detecting quasi degenerate particles is to look for events where these particles are produced associated with hard initial state radiation. These events are sensitive to the monojet search which requires large missing energy recoiling against one or two energetic jets (See figure 4.). In refs. [21-24, 38] the sensitivity of the monojet search to the higgsino-like LSP scenario has been studied. The results found in these literature vary because their simulation set up and selection cuts are different. The authors of [38] found the most optimistic results, albeit with very aggressive cuts. We use their result because they presented the expected sensitivities at the $13 \mathrm{TeV}$ LHC as functions of $m_{\tilde{\chi}_{1}^{0}}$ and $\Delta m^{ \pm}$, which enables us to translate their limit on our parameter plane. We project their 2- $\sigma$ regions into our $\left(|\mu|, M_{1}\right)$ parameter plane in figure 5 , where the darker (lighter) green region corresponds to the $100(3000) \mathrm{fb}^{-1}$ integrated luminosity. The monojet search is sensitive only for the $\Delta m^{ \pm}<20-30 \mathrm{GeV}$. Therefore the sensitivity dies off in the $|\mu| \simeq M_{1}$ region because $\Delta m^{ \pm}$becomes too large. The LHC sensitivity (green) regions are similar for other combinations of the relative signs of the parameters and all values of $\tan \beta$. They depend very weakly on the value on $M_{2}$. We can see some complementarity between the DM direct detection experiments and the collider experiments, especially for the $M_{2}$ decoupled case in figure 5. For negative values of $\mu$, there remain, unfortunately, some regions of the parameters not accesible neither at the LHC nor in the DD experiments.

\section{Wino-higgsino LSP}

The above discussion can be easily extended to neutralinos that are mainly a mixture of higgsino and wino (we take $M_{1}=7 \mathrm{TeV}$ ), which are less explored in the literature (see, however, $[19,26,27,34])$. The annihilation cross section for pure wino $\tilde{W}$ is determined by the vertex $\tilde{W}^{0} \tilde{W}^{ \pm} W^{\mp}$. One has approximately [15]

$$
\Omega_{\tilde{W}} h^{2}=0.13\left(\frac{M_{2}}{2.5 \mathrm{TeV}}\right)^{2} .
$$

This formula does not include the Sommerfeld enhancement for the wino component $[74,75]$. It is not included in the micrOMEGAs code used by us, but this does not affect the main conclusions of the paper. ${ }^{11}$ The annihilation of winos is more efficient than that of higgsinos. The minimal value of $\Omega_{\chi} h^{2}$ (for $M_{2}=100 \mathrm{GeV}$ ) reaches $2 \cdot 10^{-4}$ and the value of 0.12 is reached for $M_{2} \approx 2.3 \mathrm{GeV}$. This is seen in the left plots of figure 6 , for $\tan \beta=2, \mu<0$ and $\tan \beta=10$ and $\mu>0$. An admixture of higgsinos increases the value of the neutralino relic abundance. The values of $\Omega_{\chi} h^{2}$, when shown as a function of the

\footnotetext{
${ }^{11}$ We have confirmed this by studying several representative points with the numerical package [76] which is an extended version of DarkSUSY [77] that calculates Sommerfeld enhancement in MSSM. We thank A. Hryczuk for useful correspondence about the usage of that package.
} 

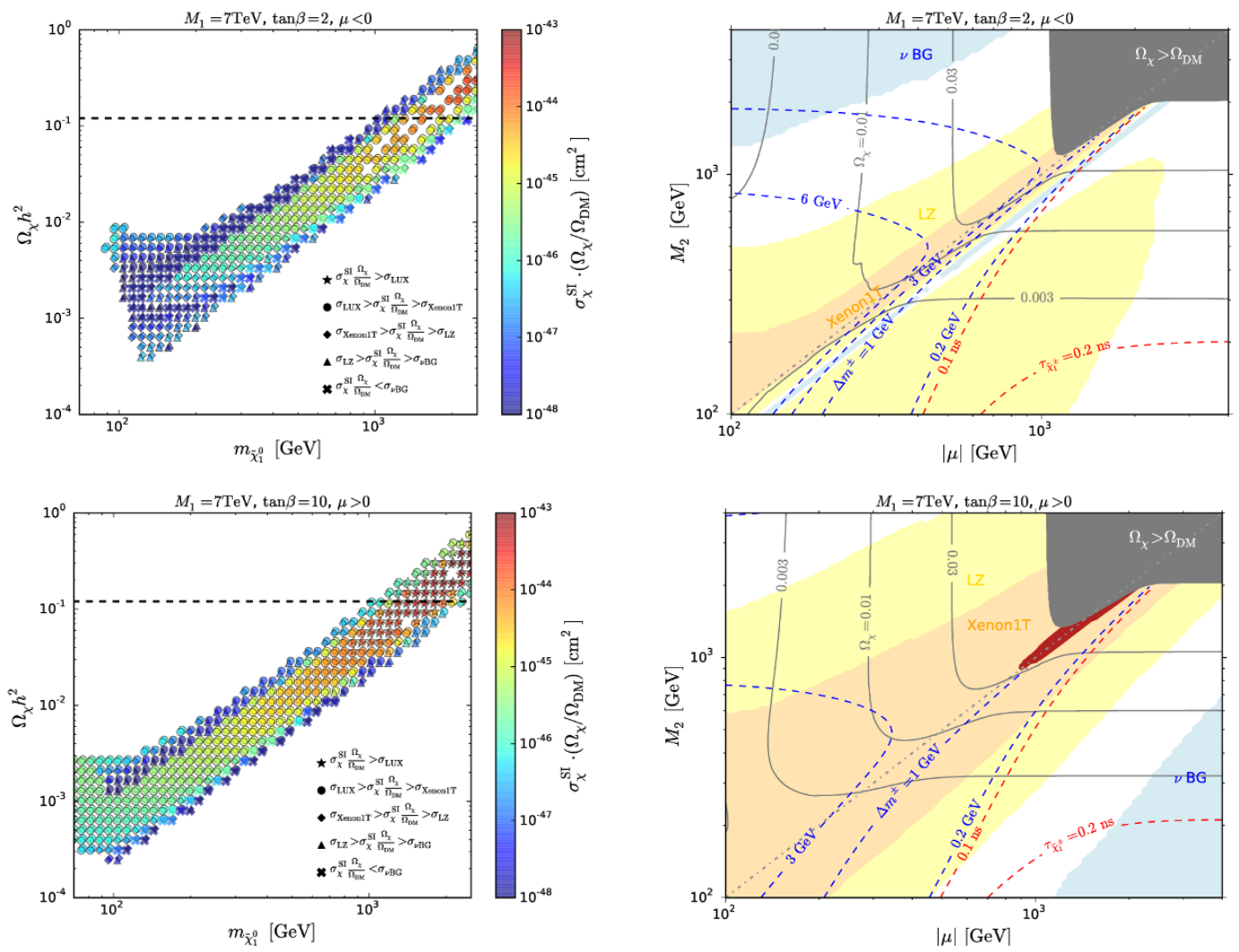

Figure 6. Same as in figure 1 but for the scan over $\mu$ and $M_{2}$, with decoupled binos.

LSP mass, fall in the region between the two boundaries given by the pure higgsino and wino states.

The tree level spin independent scattering cross section vanishes for pure higgsino and wino states. The $h \chi \chi$ coupling is approximately given by the equations (2.3), (2.4) and (2.5), with the following replacements: $M_{1} \rightarrow M_{2}, g_{1} \rightarrow g_{2}, \sin \theta_{W} \rightarrow \cos \theta_{W}$ and with the change of the sign of the first term in eq. (2.5).

Those equations explain the qualitative patterns of the results of the scan plotted in the $\left(|\mu|, M_{2}\right)$ plane shown in the right column of figure $6 .{ }^{12}$ In general, the $\sigma_{\chi}^{\mathrm{SI}}$ decreases in the direction perpendicular to the diagonal. There is an interesting dependence on $\tan \beta$ and on the sign of $\mu$. One effect is again the existence of blind spots for small $\tan \beta$ and negative $\mu$ and a strong enhancement of the spin independent scattering cross sections for positive $\mu$. For $\tan \beta=2$ and $\mu>0$ (not shown in the figure) a large part of the parameter space near the diagonal $\left|M_{2}\right| \approx|\mu|$ is already excluded by the LUX experiment. For all combinations of $\tan \beta$ and signs of $\mu$ there can be seen certain asymmetries in the values of the $\sigma_{\chi}^{\mathrm{SI}}$ with respect to the diagonal $\left|M_{2}\right| \approx|\mu|$. They can be understood by looking at the equations (2.3), (2.4) and (2.5), updated to the present case. For instance, for large $\tan \beta$ the $\sigma_{\chi}^{\mathrm{SI}}$ is larger for $|\mu|<\left|M_{2}\right|$ (dominantly higgsino) than for $\left|M_{2}\right|<|\mu|$ (dominantly

\footnotetext{
${ }^{12}$ We scan over $\left(|\mu|,\left|M_{2}\right|\right)$, both in the range $(0.1-5 \mathrm{TeV})$.
} 

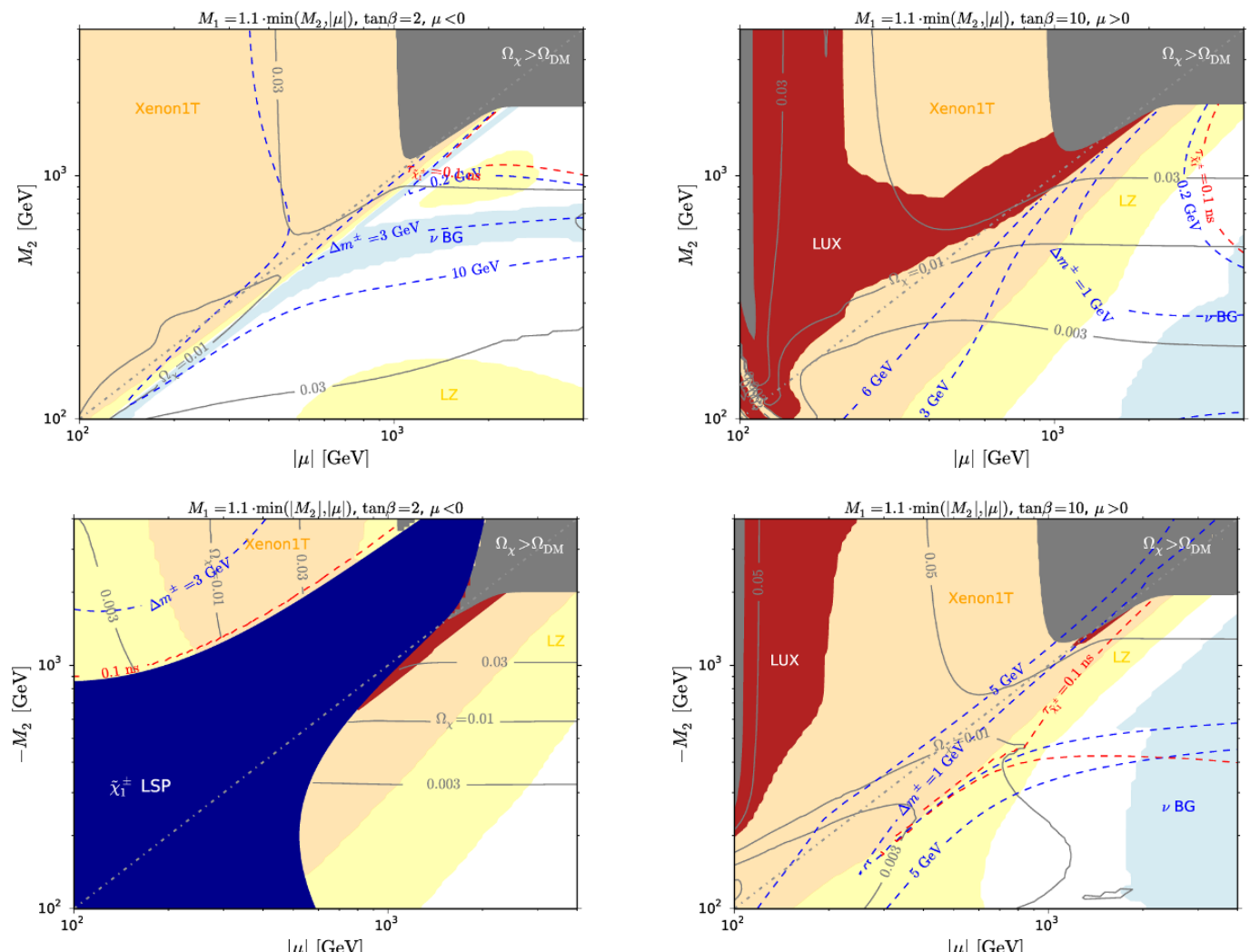

Figure 7. Same as figure 6 but for non-decoupled binos. The values of $M_{1}$ are marked on the plots.

wino) because of the $\frac{1}{M_{2}}$ versus $\frac{1}{\mu^{2}}$ suppression of the couplings in eqs. (2.4) and (2.3), respectively. There is also some residual dependence on the sign of $\mu$ coming from the numerator in eqs. (2.3) and (2.4). For small $\tan \beta$ and negative $\mu$ the asymmetry with respect to the diagonal is reversed, as can be seen from the interplay of the numerators and denominators in eqs. (2.3) and (2.4). The important conclusion is that the vast parameter range is accessible in the DD experiments. However, it is clear that the dominantly wino or higgsino LSP regions will not be reachable in the DD experiments and these regions still take up sizeable portion of the $\left(\mu, M_{2}\right)$ plane, particularly in the dominantly wino case.

The separation into the two regions, $|\mu|<\left|M_{2}\right|$ and $\left|M_{2}\right|<|\mu|$, is important for understanding the pattern of mass differences and the NLSP lifetime. The higgsino-like LSP region $\left(|\mu|<\left|M_{2}\right|\right)$ is qualitatively similar to the bino-higgsino mixed LSP region discussed in the previous section. We observe that $\Delta m^{ \pm}$is also $\mathcal{O}(1-10) \mathrm{GeV}$ but slightly larger than in the bino-higgsino case, because of the $\cos \theta_{W}^{2} / M_{2}$ corrections versus $\sin \theta_{W}^{2} / M_{1}$ corrections in the previous case (see eq. (A.5)). Also, as before, $\Delta m^{0} \approx 2 \times \Delta m^{ \pm}$.

In the wino-like LSP region $\left(\left|M_{2}\right|<|\mu|\right)$ the tree level $\Delta m^{ \pm}$vanishes up to very small corrections (see eq. (A.34) and (A.35) in the appendix) and $\Delta m^{ \pm} \approx 160-170 \mathrm{MeV}$ is given by the loop effects [78]. The lifetime becomes large, of the order of $\mathcal{O}(0.1)$ ns. On the other hand, there can be arbitrarily large mass difference $\Delta m^{0} \approx|\mu|-\left|M_{2}\right|$. 


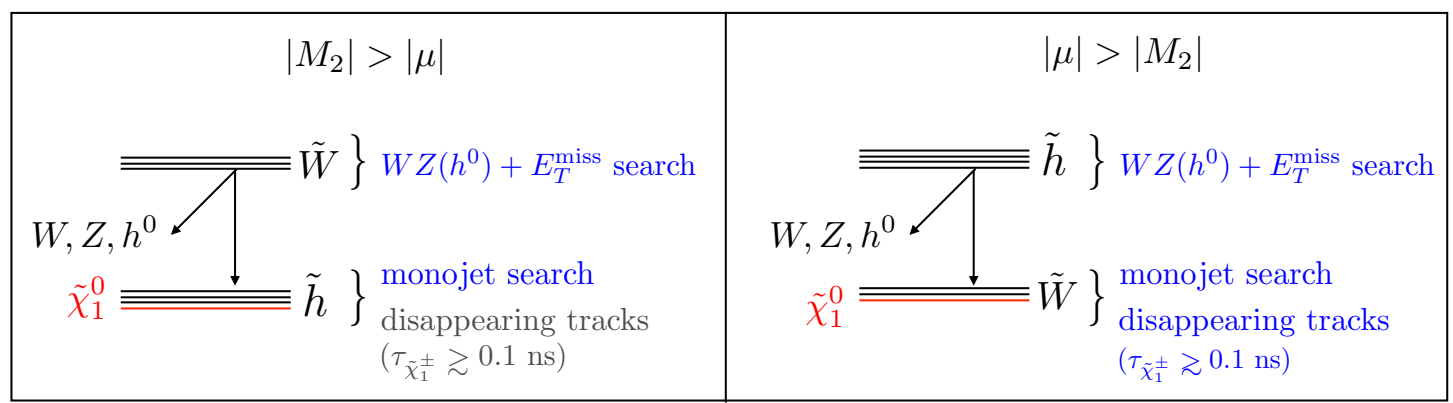

Figure 8. Strategy for collider search.

The effects of non-decoupling of $M_{1}$ in the scan over $M_{2}$ and $\mu$ are illustrated in figure 7 . One can see, comparing figure 7 with figure 6 , that the main patterns are quite similar to the decoupled case. In particular, this is true for the mass differences $\Delta m^{0}$ (not shown in the figures) and $\Delta m^{ \pm}$but the $\sigma_{\chi}^{\mathrm{SI}}$ is generically larger, especially for $\left|M_{2}\right|>|\mu|$, making easier the detection of the LSP in the future DD experiments. Adding the bino component makes the values of $\Omega_{\chi} h^{2}$ for the same neutralino mass somewhat larger, going above the higgsino bound seen in figure 6 .

\subsection{Collider}

For the wino-higgsino scenario the strategy of collider search has new elements as compared to the bino-higgsino case. Both wino-like and higgsino-like states can have sizable production cross section. For sufficiently large splitting in the values of $M_{2}$ and $\mu$, the heavier of the wino-like or the higgsino-like states can decay to the higgsino-like or winolike states, respectively, accompanied by $W, Z$ or $W, h^{0}$ boson pairs (see figure 8 ). An interesting signature for $\left|M_{2}\right|>|\mu|$ is then the decay $\tilde{W}^{ \pm} \tilde{W}^{0} \rightarrow W^{ \pm} Z(h)+E_{T}^{\text {miss }}$, where $\tilde{W}^{ \pm}$and $\tilde{W}^{0}$ are the wino-like $\tilde{\chi}_{2}^{ \pm}$and $\tilde{\chi}_{3}^{0}$, respectively and $E_{T}^{\text {miss }}$ includes higgsino-like $\tilde{\chi}_{1}^{ \pm}$, $\tilde{\chi}_{2}^{0}$ and $\tilde{\chi}_{1}^{0}$, neglecting soft particles arising from the $\tilde{\chi}_{1}^{ \pm}$and $\tilde{\chi}_{2}^{0}$ decays into an almost mass degenerate $\tilde{\chi}_{1}^{0}$. Similarly, for $|\mu|>\left|M_{2}\right|$, we have the decays $\tilde{h}^{ \pm} \tilde{h}^{0} \rightarrow W^{ \pm} Z(h)+E_{T}^{\text {miss }}$ and in this case, $\tilde{h}^{0}$ and $\tilde{h}^{ \pm}$are higgsino-like $\tilde{\chi}_{2}^{0}, \tilde{\chi}_{3}^{0}$ and $\tilde{\chi}_{2}^{ \pm}$, respectively, and $E_{T}^{\text {miss }}$ includes wino-like $\tilde{\chi}_{1}^{ \pm}$and $\tilde{\chi}_{1}^{0}$ assuming the decay products of the $\tilde{\chi}_{1}^{ \pm}$decay does not contribute to the signal regions in the analysis. Such $W+Z(h)+E_{T}^{\text {miss }}$ signatures depend on the production cross sections of the heavy gaugino/higgsino pairs and on their branching ratios into those final states. Using the Goldstone equivalence theorem [79] one can estimate $[31,32,79]$ that the branching ratio of each initial particle into $W Z(h)$ is $50 \%$, with $25 \%$ for $Z$ and $h$ each, so that we have $50 \%$ probability for the $W+Z(h)+E_{T}^{\text {miss }}$ signature and the remaining $50 \%$ goes into $W W, Z Z$ and $h h$ final states. There is no dedicated analysis of the LHC discovery potential for such signatures (such an analysis for $100 \mathrm{TeV}$ colliders is presented in [32]). CMS have studied the projected sensitivity in the $14 \mathrm{TeV}$ LHC with $3000 \mathrm{fb}^{-1}$ for the $\tilde{\chi}_{1}^{ \pm} \tilde{\chi}_{2}^{0} \rightarrow\left(W^{ \pm} \tilde{\chi}_{1}^{0}\right)\left(Z \tilde{\chi}_{1}^{0}\right)$ and the $\tilde{\chi}_{1}^{ \pm} \tilde{\chi}_{2}^{0} \rightarrow\left(W^{ \pm} \tilde{\chi}_{1}^{0}\right)\left(h^{0} \tilde{\chi}_{1}^{0}\right)$ processes [80], assuming wino-like production cross sections for the initial states and $100 \%$ $W+Z(h)+E_{T}^{\text {miss }}$ signature. In this study, $5-\sigma$ sensitivities for three different models, $\operatorname{BR}\left(\tilde{\chi}_{2}^{0} \rightarrow\left[Z \tilde{\chi}_{1}^{0}, h \tilde{\chi}_{1}^{0}\right]\right)=(100,0),(0,100),(50,50) \%$, are presented and they are found to 

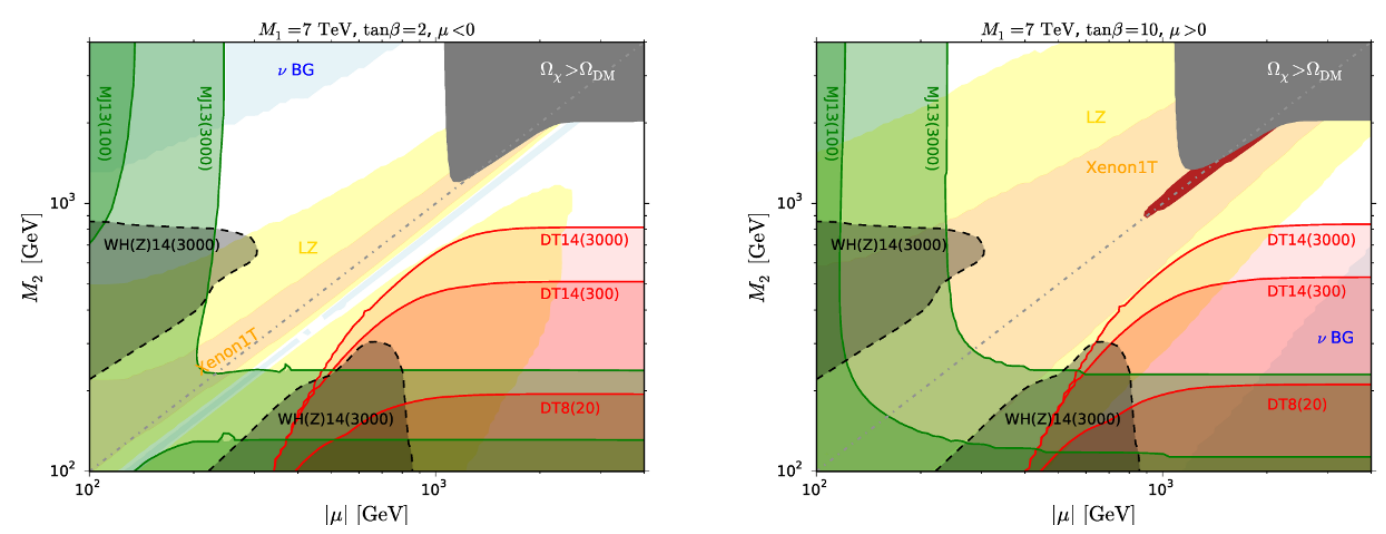

Figure 9. The LHC sensitivity regions for decoupled binos in the $\left(|\mu|, M_{2}\right)$ plane: the $W Z+E_{T}^{\text {miss }}$ and $W h+E_{T}^{\text {miss }}$ combined search (grey), the monojet search (green) and the disappearing track search (red).
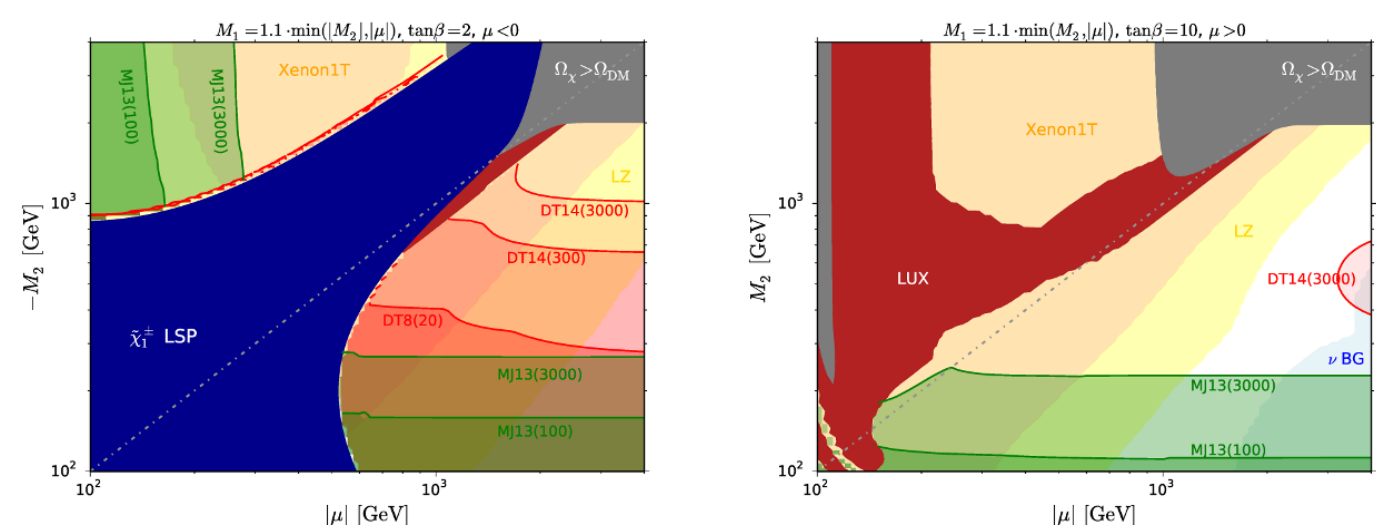

Figure 10. The LHC sensitivity regions for non-decoupled binos in the $\left(|\mu|,-M_{2}\right)$ (left) and $\left(|\mu|, M_{2}\right)$ (right) planes: the monojet search (green) and the disappearing track search (red).

be similar to each other. We can use those results for the purpose of a qualitative illustration of a ballpark sensitivity to our $\left(|\mu|, M_{2}\right)$ plane, keeping in mind that the CMS assumptions about the production cross sections and the decay signature are not strictly valid in our case, but just up to a factor of 2-3. We map the CMS 5- $\sigma$ sensitivity for the $\operatorname{BR}\left(\tilde{\chi}_{2}^{0} \rightarrow\left[Z \tilde{\chi}_{1}^{0}, h \tilde{\chi}_{1}^{0}\right]\right)=(50,50) \%$ case (in accordance with the Goldstone equivalence theorem) by identifying $m_{\tilde{\chi}_{1}^{ \pm}}^{\mathrm{CMS}}=M_{2}, m_{\tilde{\chi}_{1}^{0}}^{\mathrm{CMS}}=|\mu|$ for $M_{2}>|\mu|$, and $m_{\tilde{\chi}_{1}^{ \pm}}^{\mathrm{CMS}}=|\mu|, m_{\tilde{\chi}_{1}^{0}}^{\mathrm{CMS}}=M_{2}$ for $|\mu|>M_{2}$. The result is shown by the grey regions in figure 9 surrounded by the dashed curves. One should stress again that this is only a qualitative illustration of "an order of magnitude" for the expected sensitivity, because of the mentioned above simplified CMS assumptions about the production and decay rates, inadequate for our case.

As we discussed in section 2, the production of the lighter multiplet (lighter of the wino- or higgsino-like states) can be probed by the monojet search and the disappearing track search. In figure 9 and 10 we show the 2- $\sigma$ sensitivity region found in [38] using the same procedure as in figure 5. Since the study of [38] assumes the higgsino-like LSP 
scenario, the green region in the wino-like LSP region should be regarded as conservative since the wino cross section is larger than the higgsinos at the same mass, although we believe it is not too conservative by the same reason mentioned above.

It is known that the lifetime of charged wino can be as long as the collider scale in the almost pure wino region. In figure 6 the charged wino lifetime is longer than 0.1 ns to the right of the black dashed curves, where the search exploiting the disappearing tracks can be sensitive. ATLAS [81] and CMS [82] have looked for disappearing tracks in the $8 \mathrm{TeV}$ proton-proton collision data and identified the 95\% CL excluded regions in the $\left(m_{\tilde{\chi}_{1}^{ \pm}}, \Delta m^{ \pm}\right)$plane. Since we can compute $m_{\tilde{\chi}_{1}^{ \pm}}$and $\Delta m^{ \pm}$at each point in the $\left(|\mu|, M_{2}\right)$ plane, we can map these $95 \%$ CL excluded regions onto our parameter plane. We translate the ATLAS excluded region (figure 6 of [81]) in our $\left(|\mu|, M_{2}\right)$ plane, which are shown by the darkest red regions. We also project the $8 \mathrm{TeV}$ excluded regions into the $14 \mathrm{TeV} \mathrm{LHC}$ using Collider Reach program [83] assuming the projected limit is obtained with the same number of signal events after the event selection as observed at the $8 \mathrm{TeV}$ exclusion boundary. ${ }^{13}$ The projected sensitivity for the $14 \mathrm{TeV}$ LHC with $300(3000) \mathrm{fb}^{-1}$ is shown by the darker (light) red region.

\section{Bino-wino}

In the limit of a decoupled $\mu$, the picture is very simple because there is no mixing between the binos and winos except $M_{1}=M_{2}$. Without the bino-wino mixing, the heavier of the bino and wino cannot decay into lighter one if scalars are decoupled [85]. The lifetime of the heavier EW gaugino exceeds $c \tau \gtrsim \mathcal{O}(1) \mathrm{cm}$ with $|\mu| \gtrsim \mathcal{O}\left(10^{2-6}\right) \mathrm{TeV}$, depending on the bino-wino mass splitting.

For $\left|M_{2}\right|<M_{1}$ the LSP and the NLSP are neutral and charged winos, respectively. The LSP relic abundance is that of a pure wino, already discussed in the previous section. The mass difference $\Delta m^{ \pm}$is about $160-170 \mathrm{MeV}$, given by the loop effects and the NLSP decay has a collider scale lifetime, $c \tau_{\tilde{\chi}_{1}^{ \pm}} \sim 5-10 \mathrm{~cm}$. The mass difference $\Delta m^{0}$ is fixed by the difference $M_{1}-\left|M_{2}\right|$. The spin-independent scattering cross sections are very small, given by loop effects and below the neutrino background. The region $M_{1}<\left|M_{2}\right|$ is excluded by the bound $\Omega_{\chi} h^{2}<0.12$. The above simple picture is a good reference frame for discussing what happens when the values of the $|\mu|$ parameter come closer to the values of $M_{1}$ and/or $\left|M_{2}\right|$ (see also [21]). In figure 12 and in figure 11 we show the plots for $|\mu|=1.1 \max \left(M_{1},\left|M_{2}\right|\right)$ and for $|\mu|=1.1 \min \left(M_{1},\left|M_{2}\right|\right)$, respectively, for $\tan \beta=10, \mu>0$ and positive $M_{2}$. For the other combinations of the values of $\tan \beta$ and the signs of $\mu$ and $M_{2}$ the results are very similar.

In the scan exemplified by figure 12 the higgsino is heavy, it mediates the bino-wino mixing but its admixture remains small. In figure 11 the higgsino component in the LSP

\footnotetext{
${ }^{13}$ This is commonly used assumption. For the different energy and luminosity, the cross section, the optimal event selection and its acceptance for signal and background change. However, the signal yield at the exclusion boundary usually do not change much after optimising the selection cuts. This is an empirical observation but good agreement is often found between the results from this approach and from the full simulation. See e.g. [32, 84].
} 

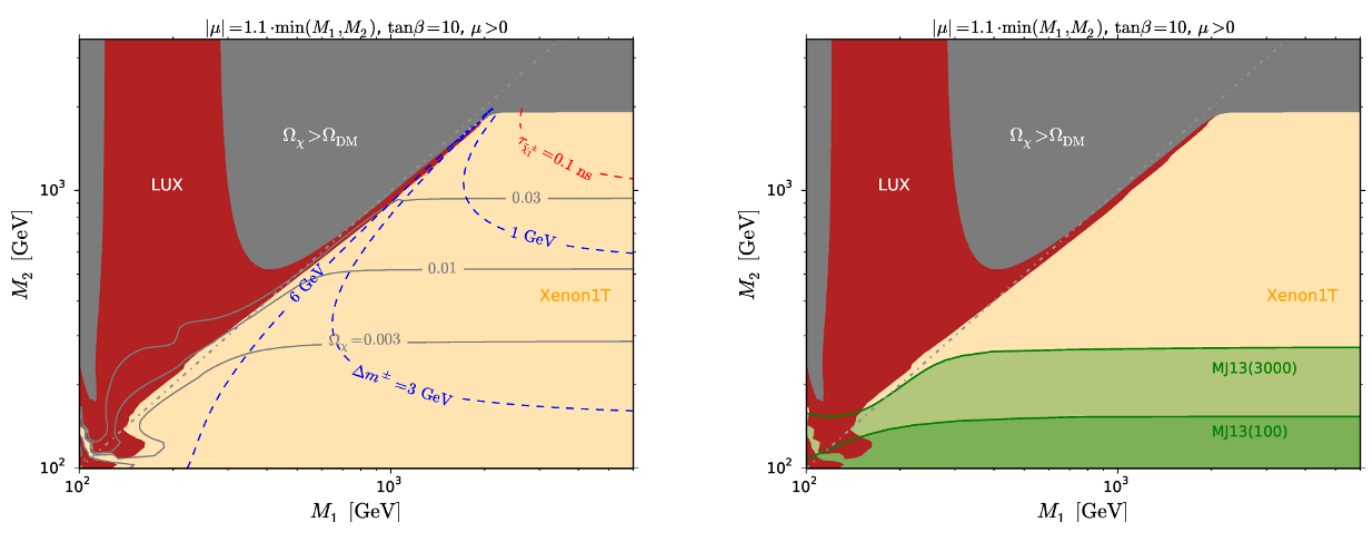

Figure 11. The results of the scan over $M_{1}$ and $M_{2}$ with $\mu$ fixed at the values marked on the plots. In the right plot, the estimated LHC discovery limit is shown.

plays already important role and the observed patterns can be understood by comparing with the plots for $10 \%$ degeneracies discussed in the previous section.

$\left|\boldsymbol{M}_{\mathbf{2}}\right|>\boldsymbol{M}_{\mathbf{1}}$. We see in figure 12 that most of the bino-dominated region is still excluded by the bound $\Omega_{\chi} h^{2} \leq 0.12$. However, for $M_{1} \approx M_{2}$ an acceptable region opens up, although for $\mu>0$ mostly excluded by the LUX limits. For $\mu<0$ (not shown in the paper), in that well-tempered bino-wino region the spin-independent cross sections are often within the reach of the Xenon1T experiment. The recent result of a general 10 parameter MSSM scan, including the $g-2$, the collider constraints as well as the DM constraints with $\Omega_{\chi}=\Omega_{D M}$, also prefers this parameter region [86, 87].

With $|\mu|=1.1 M_{1}$ (see figure 11) the LSP becomes a mixture of all three components, with large higgsino-bino mixing, and only a part of the previously excluded region is still excluded by the bound $\Omega_{\chi} h^{2} \leq 0.12$. This region resembles the regions with $M_{1}=1.1|\mu|$ in figure 7 , that is the regions above the diagonal. The regions excluded by the overproduction of the neutralinos are, however, larger because of the stronger bino component. The spin independent scattering cross sections are large and the mass differences are of order $\mathcal{O}(10) \mathrm{GeV}$.

$\left|\boldsymbol{M}_{\mathbf{2}}\right|<\boldsymbol{M}_{\mathbf{1}}$. For $|\mu|=1.1 M_{1}$, the LSP remains dominantly wino-like but some admixture of bino makes the spin-independent cross sections partly accessible in the DD experiments. The mass differences are very small and the NLSP is long-lived, with strong implications for collider searches.

With $|\mu|=1.1\left|M_{2}\right|$, the LSP is dominantly wino-higgsino. The patterns seen in figure 11 resemble those for $M_{2}=1.1|\mu|$ in figure 3 (i.e. above the diagonal), although the mass differences are typically smaller, closer to the pure wino case. The scattering cross section is below the LUX bound but above the neutrino bound.

In the right plots of figure 12 and 11 we show the estimated LHC discovery limits. We plot again the 2- $\sigma$ sensitivity of the monojet search [38] in the $\left(M_{1}, M_{2}\right)$ plane with the dark (light) green region corresponding to the $13 \mathrm{TeV}$ LHC with $100(3000) \mathrm{fb}^{-1}$ of the luminosity. The projected sensitivity for the $14 \mathrm{TeV}$ LHC with $300(3000) \mathrm{fb}^{-1}$ to the 

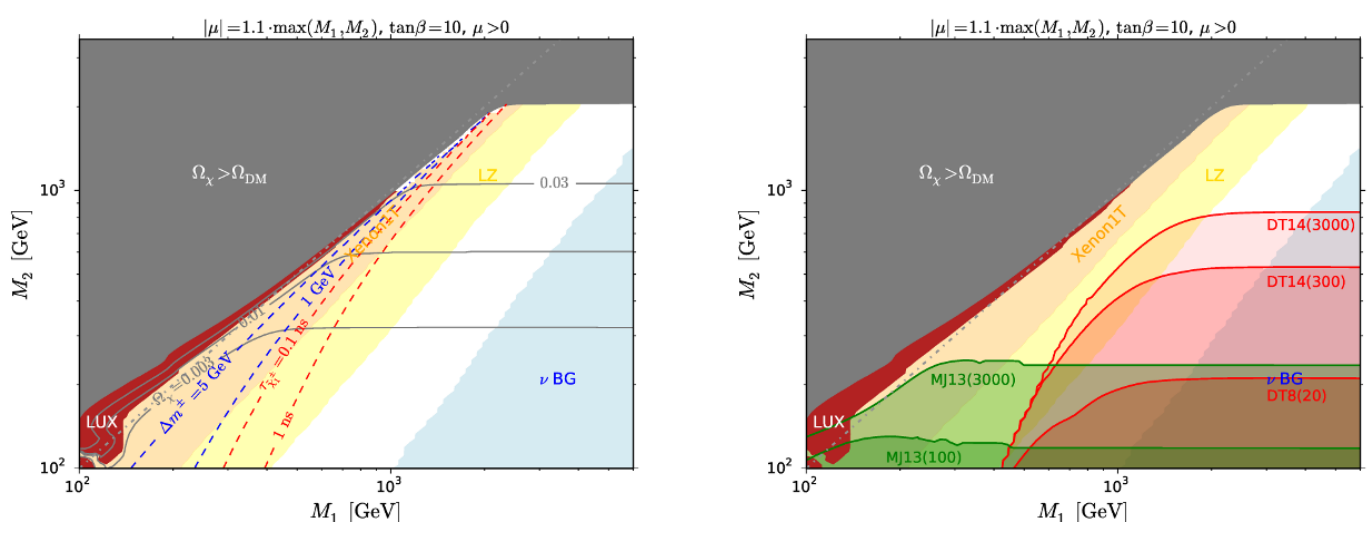

Figure 12. The results of the scan over $M_{1}$ and $M_{2}$ with $\mu$ fixed at the values marked on the plots. In the right plot, the estimated LHC discovery limit is shown.

disappearing track search, estimated as described in the previous section, is shown by the darker (light) red region.

Both, for $|\mu|=1.1 \max \left(M_{1},\left|M_{2}\right|\right)$ and $|\mu|=1.1 \min \left(M_{1},\left|M_{2}\right|\right)$, the bino-like LSP region $\left(\left|M_{2}\right|>M_{1}\right)$ is mostly excluded because of the overproduction of the neutralinos. In the other half of the parameter space $\left(M_{1}>\left|M_{2}\right|\right)$, for $|\mu|=1.1 \max \left(M_{1},\left|M_{2}\right|\right)$ the LSP is composed dominantly of wino, with some bino components. As seen in figure 12 the DD and the collider searches are complementary to each other and cover a large part of that parameter space.

With $|\mu|=1.1 \min \left(M_{1},\left|M_{2}\right|\right)$, the lighter chargino cannot have long enough lifetime to contribute to the disappearing track signature and the only channel which can probe the wino production events would be the monojet search. As seen in figure 11, for strong enough wino component the LHC can detect such production events, which will be an important cross check with the direct detection experiments such as XENON1T and LZ.

\section{$5 \quad$ Final comments and conclusions}

In the previous sections we have presented the results of several scans over certain ranges of parameters, which together cover essentially the whole relevant parameter space of the MSSM-like electroweak sector. Two conclusions have emerged from that study. One is that the two DD experiments, XENON1T and LZ, will be sensitive to neutralinos (LSPs) in almost the entire range of neutralino masses and compositions allowed by the bound $\Omega_{\chi} h^{2} \leq 0.12$. There are some exceptions, though. One to be mentioned are some regions with negative values of $\mu$, particularly for small $\tan \beta$, with a dominant higgsino component in the LSP, where some "blind spot" regions remain. This is also true for the LSP with a dominant wino component, irrespectively of the sign of $\mu$. The second conclusion is that in the electroweakino spectrum, $\Delta m^{ \pm}$(defined as the mass difference between the lighter chargino and the LSP) is smaller than 30 (10) GeV after imposing the LUX (expected from XENON1T) bounds on the neutralino spin independent scattering cross sections on nuclei. One should note however that, again, the small $\tan \beta$ and $\mu<0$ is the exception, 

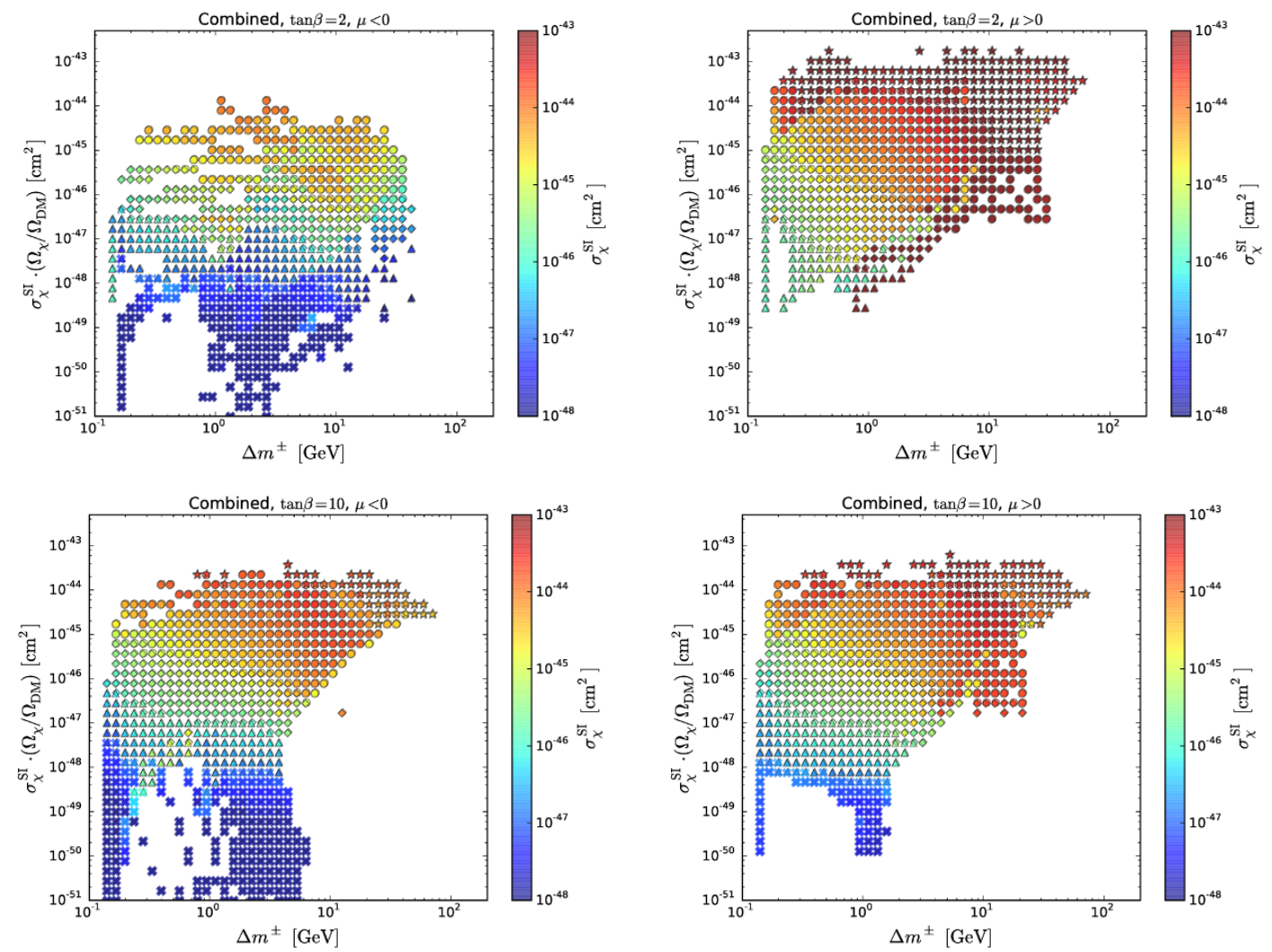

Figure 13. The combined results of the scans over $M_{1}, M_{2}$ and $\mu$ for some examples of $\tan \beta$ values and signs of $\mu$. The stars, circles, triangles, diamonds and crosses are defined in figure 1

as the mass differences can remain up to $40 \mathrm{GeV}$ even after the XENON1T and LZ results. Some of the "blind spots" regions are accessible at the LHC but not all of them. To make the generic character of those conclusions even more manifest, we show in figure 13 the combined results of the scans for a given value of $\tan \beta$ and a given sign of $\mu$.

So far, we have shown the results obtained with both slepton and squark masses set to $7 \mathrm{TeV}$. It is interesting to check how much our conclusions change when the sfermions are lighter, particularly when the sleptons are lighter. It turns out that they remain valid still when the sleptons (and of course the squarks, too) are $20 \%$ heavier than the LSP (but not lighter than $1 \mathrm{TeV}$ ). This is illustrated in figure 14, which shows the results of a scan over $|\mu|, M_{1}$ with $m_{\tilde{\ell}}=\max \left(1 \mathrm{TeV}, 1.2 \min \left(M_{1},|\mu|\right)\right)$. Indeed, figure 14 and figure 1 are very similar.

Finally, we comment on the collider searches. We have presented rough estimates of the LHC sensitivity, based on the previous results of ref. [38] for the monojet search, ref. [80] for $\tilde{\chi}_{1}^{ \pm} \tilde{\chi}_{2}^{0} \rightarrow\left(W^{ \pm} \tilde{\chi}_{1}^{0}\right)\left(Z\left(h^{0}\right) \tilde{\chi}_{1}^{0}\right)$ search and ref. [81] for the disappearing track search. A more dedicated analysis focused on the parameter range of interest emerging from the present paper would certainly be worthwhile. It should include a search for new interesting channels and techniques. For instance, some studies at $13 \mathrm{TeV}$ LHC [29] and a $100 \mathrm{TeV}$ [33] $p p$ collider have been done to attack the compressed spectra that appear in 

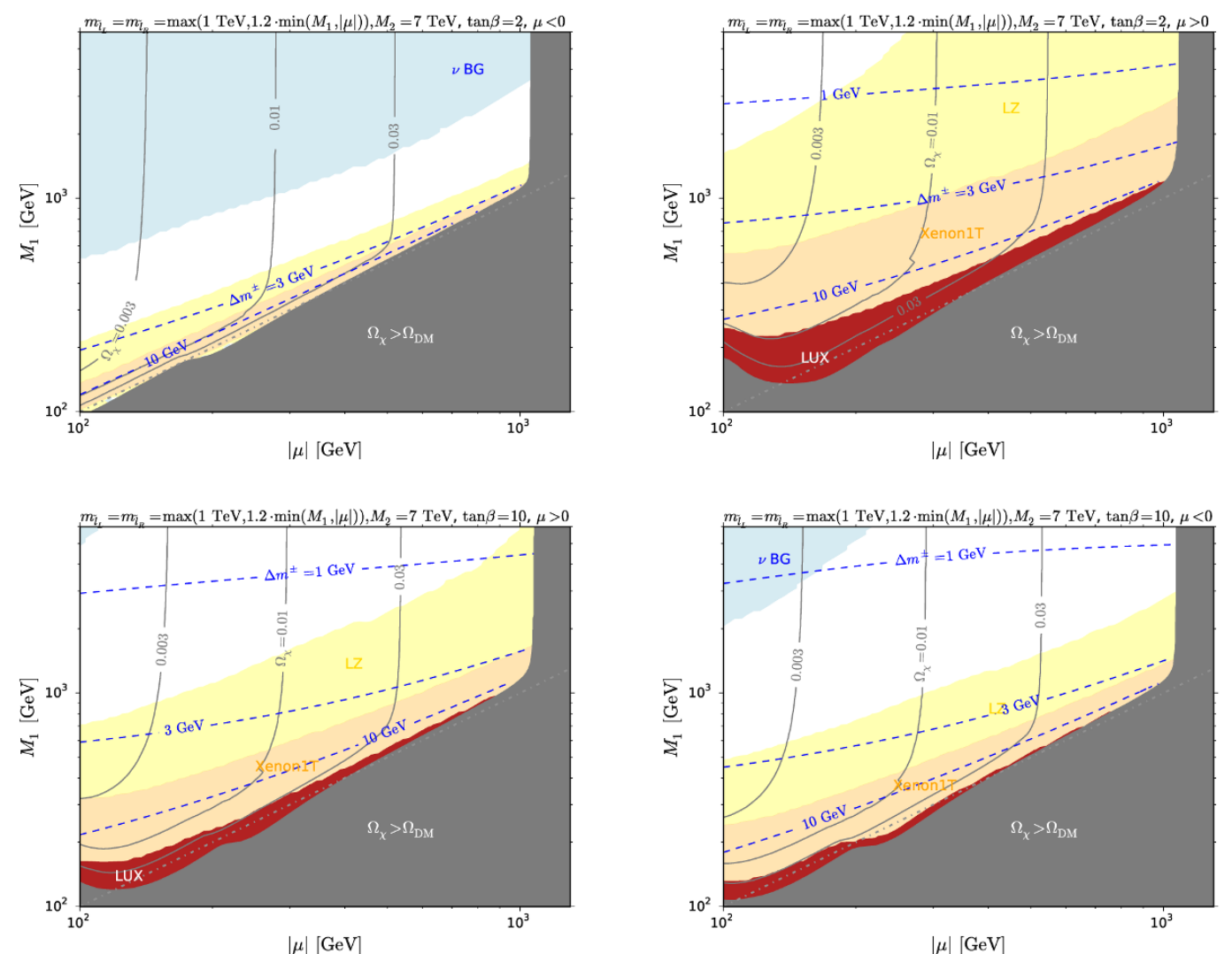

Figure 14. The results of the scan over $M_{1}, \mu$ with sleptons lighter than squarks
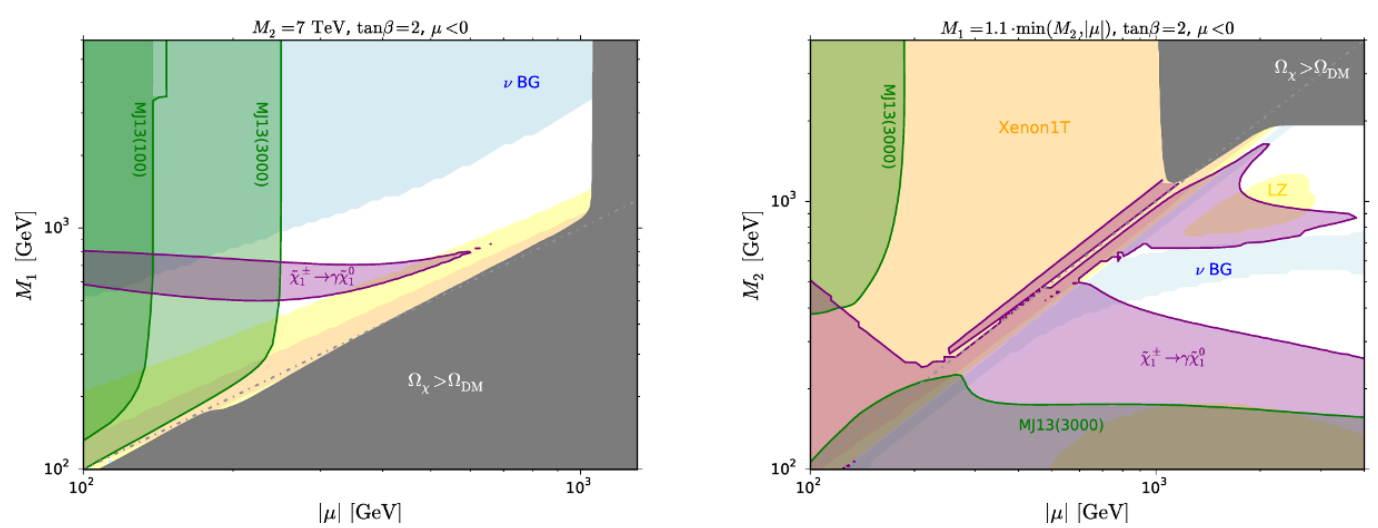

Figure 15. The region of potential sensitivity to the $\tilde{\chi}_{2}^{0} \rightarrow \gamma \tilde{\chi}_{1}^{0}$ decay is shown by purple.

the higgsino-like LSP scenarios. The idea is to look for a soft dilepton plus a soft photon originated from $\tilde{\chi}_{1}^{ \pm} \rightarrow W^{*} \tilde{\chi}_{1}^{0}$ and $\tilde{\chi}_{2}^{0} \rightarrow \gamma \tilde{\chi}_{1}^{0}$ decays, respectively. Here we simply show in figure 15 by purple an example of the region with $\Delta m^{0}>3 \mathrm{GeV}$ and $\operatorname{Br}\left(\tilde{\chi}_{2}^{0} \rightarrow \gamma \tilde{\chi}_{1}^{0}\right)>0.1{ }^{14}$ This region is potentially sensitive to more extensive analysis dedicated to this channel.

\footnotetext{
${ }^{14} \operatorname{Br}\left(\tilde{\chi}_{1}^{ \pm} \rightarrow W^{*} \tilde{\chi}_{1}^{0}\right) \sim 1$ across our $\left(|\mu|, M_{1}\right)$ parameter plane.
} 
The overall conclusion of this paper is that the electroweakino sector, being subject to important physical constraints, is a very promising territory in search for supersymmetry. In some regions of the parameter space the DD and the LHC experiments have comparable discovery potential and supplement each other and in some other regions they are complementary to each other. The collider discovery potential would significantly improve if new techniques were developed to search for the weakly produced states with the mass differences between the NLSP and LSP of order of $1 \mathrm{GeV}$.

\section{Acknowledgments}

We thank Malcolm Fairbairn and Marc Kamionkowski for helpful discussion. This work was partially supported by Polish National Science Centre under research grants DEC2012/05/B/ST2/02597, DEC-2014/15/B/ST2/02157 and DEC-2012/04/A/ST2/00099. The work of AD was supported in part by the National Science Foundation under Grant No. PHY-1215979. K.S. is supported in part by the London Centre for Terauniverse Studies (LCTS), using funding from the European Research Council via the Advanced Investigator Grant 267352. MB was partially supported by the Foundation for Polish Science through its programme HOMING PLUS and the MNiSW grant IP2012 030272.

\section{A Neutralino and chargino composition and masses}

In this appendix we present approximate formulae for the masses of $\chi_{1}^{0}, \chi_{2}^{0}$ and $\chi_{1}^{+}$, the composition of LSP and NLSP and the LSP-Higgs coupling in some limits. The make the formulae more compact we use the notation: $s_{\theta_{W}}=\sin \theta_{W}, c_{\theta_{W}}=\cos \theta_{W}, s_{\beta}=\sin \beta$, $s_{2 \beta}=\sin (2 \beta)$ etc.

\section{A.1 $|\mu| \ll\left|M_{1}\right|,\left|M_{2}\right|$}

The masses of the higgsino-dominated states up to terms of the second order in $1 / M_{1,2}$ are given by:

$$
\begin{aligned}
& m_{\chi_{1}^{0}} \approx|\mu|-\frac{M_{Z}^{2}}{2}\left|\left(\frac{c_{\theta_{W}}^{2}}{M_{2}}+\frac{s_{\theta_{W}}^{2}}{M_{1}}\right)+\mu s_{2 \beta}\left(\frac{c_{\theta_{W}}^{2}}{M_{2}^{2}}+\frac{s_{\theta_{W}}^{2}}{M_{1}^{2}}\right)\right| \\
&-\frac{M_{Z}^{2}}{2}\left[\operatorname{sgn}(\mu) s_{2 \beta}\left(\frac{c_{\theta_{W}}^{2}}{M_{2}}+\frac{s_{\theta_{W}}^{2}}{M_{1}}\right)+|\mu|\left(\frac{c_{\theta_{W}}^{2}}{M_{2}^{2}}+\frac{s_{\theta_{W}}^{2}}{M_{1}^{2}}\right)\right] \\
&+\frac{M_{Z}^{4}}{8|\mu|}\left(\frac{c_{\theta_{W}}^{2}}{M_{2}}+\frac{s_{\theta_{W}}^{2}}{M_{1}}\right)^{2} c_{2 \beta}^{2}, \\
& m_{\chi_{2}^{0}} \approx|\mu|+\frac{M_{Z}^{2}}{2}\left|\left(\frac{c_{\theta_{W}}^{2}}{M_{2}}+\frac{s_{\theta_{W}}^{2}}{M_{1}}\right)+\mu s_{2 \beta}\left(\frac{c_{\theta_{W}}^{2}}{M_{2}^{2}}+\frac{s_{\theta_{W}}^{2}}{M_{1}^{2}}\right)\right| \\
& \quad-\frac{M_{Z}^{2}}{2}\left[\operatorname{sgn}(\mu) s_{2 \beta}\left(\frac{c_{\theta_{W}}^{2}}{M_{2}}+\frac{s_{\theta_{W}}^{2}}{M_{1}}\right)+|\mu|\left(\frac{c_{\theta_{W}}^{2}}{M_{2}^{2}}+\frac{s_{\theta_{W}}^{2}}{M_{1}^{2}}\right)\right]
\end{aligned}
$$




$$
\begin{gathered}
+\frac{M_{Z}^{4}}{8|\mu|}\left(\frac{c_{\theta_{W}}^{2}}{M_{2}}+\frac{s_{\theta_{W}}^{2}}{M_{1}}\right)^{2} c_{2 \beta}^{2}, \\
m_{\chi_{1}^{+}} \approx \operatorname{sgn}(\mu)\left[\mu-M_{Z}^{2} \frac{c_{\theta_{W}}^{2}}{M_{2}}\left(s_{2 \beta}+\frac{\mu}{M_{2}}\right)\right] .
\end{gathered}
$$

In the same approximation the mass differences read:

$$
\begin{aligned}
m_{\chi_{2}^{0}}-m_{\chi_{1}^{0}} \approx M_{Z}^{2}\left|\left(\frac{c_{\theta_{W}}^{2}}{M_{2}}+\frac{s_{\theta_{W}}^{2}}{M_{1}}\right)+\mu s_{2 \beta}\left(\frac{c_{\theta_{W}}^{2}}{M_{2}^{2}}+\frac{s_{\theta_{W}}^{2}}{M_{1}^{2}}\right)\right| \\
m_{\chi_{1}^{+}}-m_{\chi_{1}^{0}} \approx \frac{M_{Z}^{2}}{2}\left|\left(\frac{c_{\theta_{W}}^{2}}{M_{2}}+\frac{s_{\theta_{W}}^{2}}{M_{1}}\right)+\mu s_{2 \beta}\left(\frac{c_{\theta_{W}}^{2}}{M_{2}^{2}}+\frac{s_{\theta_{W}}^{2}}{M_{1}^{2}}\right)\right| \\
-\frac{M_{Z}^{2}}{2}\left[\operatorname{sgn}(\mu) s_{2 \beta}\left(\frac{c_{\theta_{W}}^{2}}{M_{2}}-\frac{s_{\theta_{W}}^{2}}{M_{1}}\right)+|\mu|\left(\frac{c_{\theta_{W}}^{2}}{M_{2}^{2}}-\frac{s_{\theta_{W}}^{2}}{M_{1}^{2}}\right)\right] \\
-\frac{M_{Z}^{4}}{8|\mu|}\left(\frac{c_{\theta_{W}}^{2}}{M_{2}}+\frac{s_{\theta_{W}}^{2}}{M_{1}}\right)^{2} c_{2 \beta}^{2} .
\end{aligned}
$$

The composition of the LSP (NLSP) is given by

$$
\begin{aligned}
& N_{i 1} \approx-\frac{1}{\sqrt{2}} \frac{M_{Z}}{M_{1}}\left(1-(-1)^{i} \eta \frac{|\mu|}{M_{1}}\right) s_{\theta_{W}}\left(s_{\beta}-(-1)^{i} \eta \operatorname{sgn}(\mu) c_{\beta}\right) \\
& +\frac{1}{4 \sqrt{2}} \frac{M_{Z}^{3}}{|\mu| M_{1}}\left(\frac{c_{\theta_{W}}^{2}}{M_{2}}+\frac{s_{\theta_{W}}^{2}}{M_{1}}\right) s_{\theta_{W}} c_{2 \beta}\left((-1)^{i} \eta s_{\beta}+\operatorname{sgn}(\mu) c_{\beta}\right), \\
& N_{i 2} \approx+\frac{1}{\sqrt{2}} \frac{M_{Z}}{M_{2}}\left(1-(-1)^{i} \eta \frac{|\mu|}{M_{2}}\right) c_{\theta_{W}}\left(s_{\beta}-(-1)^{i} \eta \operatorname{sgn}(\mu) c_{\beta}\right) \\
& -\frac{1}{4 \sqrt{2}} \frac{M_{Z}^{3}}{|\mu| M_{2}}\left(\frac{c_{\theta_{W}}^{2}}{M_{2}}+\frac{s_{\theta_{W}}^{2}}{M_{1}}\right) c_{\theta_{W}} c_{2 \beta}\left((-1)^{i} \eta s_{\beta}+\operatorname{sgn}(\mu) c_{\beta}\right), \\
& N_{i 3} \approx \frac{\operatorname{sgn}(\mu)}{\sqrt{2}}\left[(-1)^{i} \eta+\frac{1}{4} \frac{M_{Z}^{2}}{|\mu|}\left(\frac{c_{\theta_{W}}^{2}}{M_{2}}+\frac{s_{\theta_{W}}^{2}}{M_{1}}\right) c_{2 \beta}\right. \\
& +\frac{M_{Z}^{2}}{4}\left(\frac{c_{\theta_{W}}^{2}}{M_{2}^{2}}+\frac{s_{\theta_{W}}^{2}}{M_{1}^{2}}\right)\left(\operatorname{sgn}(\mu) s_{2 \beta}-2(-1)^{i} \eta c_{\beta}^{2}\right) \\
& \left.+\frac{1}{32} \frac{M_{Z}^{4}}{\mu^{2}}\left(\frac{c_{\theta_{W}}^{2}}{M_{2}}+\frac{s_{\theta_{W}}^{2}}{M_{1}}\right)^{2} c_{2 \beta}\left(4 \operatorname{sgn}(\mu) s_{2 \beta}-(-1)^{i} \eta c_{2 \beta}\right)\right], \\
& N_{i 4} \approx \frac{1}{\sqrt{2}}\left[1-(-1)^{i} \eta \frac{1}{4} \frac{M_{Z}^{2}}{|\mu|}\left(\frac{c_{\theta_{W}}^{2}}{M_{2}}+\frac{s_{\theta_{W}}^{2}}{M_{1}}\right) c_{2 \beta}\right. \\
& -\frac{M_{Z}^{2}}{4}\left(\frac{c_{\theta_{W}}^{2}}{M_{2}^{2}}+\frac{s_{\theta_{W}}^{2}}{M_{1}^{2}}\right)\left(-(-1)^{i} \eta \operatorname{sgn}(\mu) s_{2 \beta}+2 s_{\beta}^{2}\right) \\
& \left.-\frac{1}{32} \frac{M_{Z}^{4}}{\mu^{2}}\left(\frac{c_{\theta_{W}}^{2}}{M_{2}}+\frac{s_{\theta_{W}}^{2}}{M_{1}}\right)^{2} c_{2 \beta}\left(4(-1)^{i} \eta \operatorname{sgn}(\mu) s_{2 \beta}+c_{2 \beta}\right)\right] \text {, }
\end{aligned}
$$


where $i=1,2$ and

$$
\eta=\operatorname{sgn}\left(\frac{c_{\theta_{W}}^{2}}{M_{2}}+\frac{s_{\theta_{W}}^{2}}{M_{1}}\right) .
$$

The coupling of the LSP to the Higgs scalar, in the decoupling limit, is given by

$$
c_{h \chi \chi}=\frac{1}{2}\left(g_{2} N_{12}-g_{1} N_{11}\right)\left(s_{\beta} N_{14}-c_{\beta} N_{13}\right) .
$$

Using eqs. (A.6)-(A.9) for $i=1$, and keeping terms up to the first subleading order we get

$$
c_{h \chi \chi} \approx \frac{M_{Z}}{4} \eta\left[\left|\frac{g_{2} c_{\theta_{W}}}{M_{2}}+\frac{g_{1} s_{\theta_{W}}}{M_{1}}\right|+|\mu|\left(\frac{g_{2} c_{\theta_{W}}}{M_{2}^{2}}+\frac{g_{1} s_{\theta_{W}}}{M_{1}^{2}}\right)\right]\left(1+\eta \operatorname{sgn}(\mu) s_{2 \beta}\right) .
$$

For decoupled wino this simplifies to

$$
c_{h \chi \chi} \approx \frac{g_{1} M_{Z} s_{\theta_{W}}}{4 M_{1}}\left(1+\frac{|\mu|}{\left|M_{1}\right|}\right)\left(1+\operatorname{sgn}\left(\mu M_{1}\right) s_{2 \beta}\right)
$$

which, for positive $M_{1}$ and with one higher order term added, gives eq. (2.4). Using eqs. (A.8) and (A.9) for $i=1$, one may calculate the coupling of the LSP to the $Z$ boson, $c_{Z \chi \chi}=\frac{g_{2}}{2 c_{\theta_{W}}}\left(N_{13}^{2}-N_{14}^{2}\right)$. The result, up to the first subleading order in $M_{1,2}^{-1}$, reads:

$$
c_{Z \chi \chi} \approx-\frac{g_{2} c_{2 \beta}}{4 c_{\theta_{W}}}\left[\frac{M_{Z}^{2}}{|\mu|}\left|\frac{c_{\theta_{W}}^{2}}{M_{2}}+\frac{s_{\theta_{W}}^{2}}{M_{1}}\right|+M_{Z}^{2}\left(\frac{c_{\theta_{W}}^{2}}{M_{2}^{2}}+\frac{s_{\theta_{W}}^{2}}{M_{1}^{2}}\right)+\frac{\eta}{2} s_{2 \beta} \frac{M_{Z}^{4}}{\mu|\mu|}\left(\frac{c_{\theta_{W}}^{2}}{M_{2}}+\frac{s_{\theta_{W}}^{2}}{M_{1}}\right)^{2}\right] .
$$

\section{A.2 $\left|M_{1}\right|,\left|M_{2}\right| \ll|\mu|$}

Masses of light gaugino-dominated states:

$$
\begin{aligned}
& m_{\chi_{W}^{0}} \approx\left|M_{2}-\frac{M_{Z}^{2}}{\mu} c_{\theta_{W}}^{2} s_{2 \beta}-\frac{M_{2} M_{Z}^{2}}{\mu^{2}} c_{\theta_{W}}^{2}+\frac{1}{4} \frac{M_{Z}^{4}}{\mu^{2}\left(M_{2}-M_{1}\right)} s_{2 \theta_{W}}^{2} s_{2 \beta}^{2}\right|, \\
& m_{\chi_{B}^{0}} \approx\left|M_{1}-\frac{M_{Z}^{2}}{\mu} s_{\theta_{W}}^{2} s_{2 \beta}-\frac{M_{1} M_{Z}^{2}}{\mu^{2}} s_{\theta_{W}}^{2}-\frac{1}{4} \frac{M_{Z}^{4}}{\mu^{2}\left(M_{2}-M_{1}\right)} s_{2 \theta_{W}}^{2} s_{2 \beta}^{2}\right|, \\
& m_{\chi_{1}^{+}} \approx\left|M_{2}-\frac{M_{Z}^{2}}{\mu} c_{\theta_{W}}^{2} s_{2 \beta}-\frac{M_{2} M_{Z}^{2}}{\mu^{2}} c_{\theta_{W}}^{2}\right| .
\end{aligned}
$$

When $\left|M_{1}\right|<\left|M_{2}\right|$ the LSP is bino-dominated $\chi_{B}^{0}$ and

$$
m_{\chi_{1}^{+}}-m_{\chi_{1}^{0}} \approx m_{\chi_{2}^{0}}-m_{\chi_{1}^{0}} \approx\left|M_{2}\right|-\left|M_{1}\right|+\frac{M_{Z}^{2}}{|\mu|} s_{2 \beta}\left[\operatorname{sgn}\left(M_{1}\right) s_{\theta_{W}}^{2}-\operatorname{sgn}\left(M_{2}\right) c_{\theta_{W}}^{2}\right]
$$

while for $\left|M_{2}\right|<\left|M_{1}\right|$ the LSP is wino-dominated $\chi_{W}^{0}$ and

$$
m_{\chi_{1}^{+}}-m_{\chi_{1}^{0}} \approx \frac{1}{4} \operatorname{sgn}\left(M_{1} M_{2}\right) \frac{M_{Z}^{4}}{\mu^{2}\left|M_{1}-M_{2}\right|} s_{2 \theta_{W}}^{2} s_{2 \beta}^{2} .
$$

So, this mass difference is positive (negative) when $M_{1}$ and $M_{2}$ have the same (oppositve) sign. The LSP and NLSP composition read:

$$
N_{B 1} \approx 1-\frac{1}{2} \frac{M_{Z}^{2}}{\mu^{2}} s_{\theta_{W}}^{2}-\frac{1}{8} \frac{M_{Z}^{4}}{\mu^{2}\left(M_{2}-M_{1}\right)^{2}} s_{2 \theta_{W}}^{2} s_{2 \beta}^{2},
$$




$$
\begin{aligned}
& N_{B 2} \approx-\frac{1}{2} \frac{M_{Z}^{2}}{\mu\left(M_{2}-M_{1}\right)} s_{2 \theta_{W}} s_{2 \beta}-\frac{1}{2} \frac{M_{1} M_{Z}^{2}}{\mu^{2}\left(M_{2}-M_{1}\right)} s_{2 \theta_{W}}-\frac{1}{4} \frac{M_{Z}^{4}}{\mu^{2}\left(M_{2}-M_{1}\right)^{2}} s_{4 \theta_{W}} s_{2 \beta}^{2} \\
& N_{B 3} \approx+\frac{M_{Z}}{\mu} s_{\theta_{W}} s_{\beta}+\frac{M_{1} M_{Z}}{\mu^{2}} s_{\theta_{W}} c_{\beta}+\frac{M_{Z}^{3}}{\mu^{2}\left(M_{2}-M_{1}\right)} s_{\theta_{W}} c_{\theta_{W}}^{2} s_{\beta} s_{2 \beta} \\
& N_{B 4} \approx-\frac{M_{Z}}{\mu} s_{\theta_{W}} c_{\beta}-\frac{M_{1} M_{Z}}{\mu^{2}} s_{\theta_{W}} s_{\beta}-\frac{M_{Z}^{3}}{\mu^{2}\left(M_{2}-M_{1}\right)} s_{\theta_{W}} c_{\theta_{W}}^{2} c_{\beta} s_{2 \beta} \\
& N_{W 1} \approx+\frac{1}{2} \frac{M_{Z}^{2}}{\mu\left(M_{2}-M_{1}\right)} s_{2 \theta_{W}} s_{2 \beta}+\frac{1}{2} \frac{M_{2} M_{Z}^{2}}{\mu^{2}\left(M_{2}-M_{1}\right)} s_{2 \theta_{W}}+\frac{1}{4} \frac{M_{Z}^{4}}{\mu^{2}\left(M_{2}-M_{1}\right)^{2}} s_{4 \theta_{W}} s_{2 \beta}^{2} \\
& N_{W 2} \approx 1-\frac{1}{2} \frac{M_{Z}^{2}}{\mu^{2}} c_{\theta_{W}}^{2}-\frac{1}{8} \frac{M_{Z}^{4}}{\mu^{2}\left(M_{2}-M_{1}\right)^{2}} s_{2 \theta_{W}}^{2} s_{2 \beta}^{2}, \\
& N_{W 3} \approx-\frac{M_{Z}}{\mu} c_{\theta_{W}} s_{\beta}-\frac{M_{2} M_{Z}}{\mu^{2}} c_{\theta_{W}} c_{\beta}+\frac{M_{Z}^{3}}{\mu^{2}\left(M_{2}-M_{1}\right)} s_{\theta_{W}}^{2} c_{\theta_{W}} s_{\beta} s_{2 \beta} \\
& N_{W 4} \approx+\frac{M_{Z}}{\mu} c_{\theta_{W}} c_{\beta}+\frac{M_{2} M_{Z}}{\mu^{2}} c_{\theta_{W}} s_{\beta}-\frac{M_{Z}^{3}}{\mu^{2}\left(M_{2}-M_{1}\right)} s_{\theta_{W}}^{2} c_{\theta_{W}} c_{\beta} s_{2 \beta}
\end{aligned}
$$

The approximation used for $\left|M_{1}\right|,\left|M_{2}\right| \ll|\mu|$ brakes down for very small values of the difference $\left|M_{2}-M_{1}\right|$.

The coupling to the Higgs boson is approximately given by

$$
c_{h \chi \chi} \approx \frac{g_{1}}{2} \frac{M_{Z} s_{\theta_{W}}}{\mu}\left(s_{2 \beta}+\frac{M_{1}}{\mu}\right)\left[1+\frac{M_{Z}^{2} c_{\theta_{W}}^{2}}{\mu\left(M_{2}-M_{1}\right)} s_{2 \beta}\right]
$$

for the bino-dominated LSP and

$$
c_{h \chi \chi} \approx \frac{g_{2}}{2} \frac{M_{Z} c_{\theta_{W}}}{\mu}\left(s_{2 \beta}+\frac{M_{2}}{\mu}\right)\left[1+\frac{M_{Z}^{2} s_{\theta_{W}}^{2}}{\mu\left(M_{1}-M_{2}\right)} s_{2 \beta}\right]
$$

for the wino-dominated one. The second term in the square bracket of eq. (A.28) formally gives contributions of the sub-sub-leading order. However, for small values of the difference $\left(M_{2}-M_{1}\right)$ it may lead to important numerical effects. For small $\tan \beta$ and negative (positive) product $\mu\left(M_{2}-M_{1}\right)$ this term gives quite substantial decrease (increase) of $c_{h \chi \chi}$ of the bino-dominated LSP. Analogous term in eq. (A.29) is somewhat less important for the case of the wino-dominated LSP because $s_{\theta_{W}}^{2}$ is more than 3 times smaller than $c_{\theta_{W}}^{2}$.

The coupling to the $Z$ boson is approximately equal

$$
c_{Z \chi \chi} \approx-\frac{g_{1} s_{\theta_{W}}}{2} c_{2 \beta} \frac{M_{Z}^{2}}{\mu^{2}}\left[1-\frac{M_{1}^{2}}{\mu^{2}}+\frac{2 M_{Z}^{2} c_{\theta_{W}}^{2}}{\mu\left(M_{2}-M_{1}\right)} s_{2 \beta}\right]
$$

for the bino-dominated LSP and

$$
c_{Z \chi \chi} \approx-\frac{g_{2} c_{\theta_{W}}}{2} c_{2 \beta} \frac{M_{Z}^{2}}{\mu^{2}}\left[1-\frac{M_{2}^{2}}{\mu^{2}}+\frac{2 M_{Z}^{2} s_{\theta_{W}}^{2}}{\mu\left(M_{1}-M_{2}\right)} s_{2 \beta}\right]
$$

for the wino-dominated one. 


\section{A.3 $\left|M_{2}\right| \ll|\mu|,\left|M_{1}\right|$}

The masses of the wino-dominated states are approximated by:

$$
\begin{gathered}
m_{\chi_{1}^{0}} \approx \mid M_{2}-\frac{M_{Z}^{2}}{\mu} c_{\theta_{W}}^{2} s_{2 \beta}-\frac{M_{2} M_{Z}^{2}}{\mu^{2}} c_{\theta_{W}}^{2} \\
\quad+\frac{M_{Z}^{4}}{\mu^{3}} c_{\theta_{W}}^{4} s_{2 \beta}-\frac{M_{2}^{2} M_{Z}^{2}}{\mu^{3}} c_{\theta_{W}}^{2} s_{2 \beta}-\frac{1}{4} \frac{M_{Z}^{4}}{\mu^{2} M_{1}} s_{2 \theta_{W}}^{2} s_{2 \beta}^{2} \mid, \\
m_{\chi_{1}^{+}} \approx\left|M_{2}-\frac{M_{Z}^{2}}{\mu} c_{\theta_{W}}^{2} s_{2 \beta}-\frac{M_{2} M_{Z}^{2}}{\mu^{2}} c_{\theta_{W}}^{2}+\frac{M_{Z}^{4}}{\mu^{3}} c_{\theta_{W}}^{4} s_{2 \beta}-\frac{M_{2}^{2} M_{Z}^{2}}{\mu^{3}} c_{\theta_{W}}^{2} s_{2 \beta}\right| .
\end{gathered}
$$

We keep the third order terms in the above expansions because the leading contribution to the NLSP(chargino)-LSP mass difference is of this order:

$$
m_{\chi_{1}^{+}}-m_{\chi_{1}^{0}} \approx \frac{1}{4} \operatorname{sgn}\left(M_{2}\right) \frac{M_{Z}^{4}}{\mu^{2} M_{1}} s_{2 \theta_{W}}^{2} s_{2 \beta}^{2} .
$$

As in the previous case this mass difference is positive (negative) when $M_{1}$ and $M_{2}$ have the same (oppositve) sign. But its absolute value is small so $m_{\chi_{1}^{+}}-m_{\chi_{1}^{0}}$ is dominated by the loop contribution. For totally decoupled bino the tree level mass difference of the NLSP and LSP starts at the 4-th order:

$$
m_{\chi_{1}^{+}}-m_{\chi_{1}^{0}} \approx \frac{1}{2}\left|M_{2}\right| c_{\theta_{W}}^{4} c_{2 \beta}^{2} \frac{M_{Z}^{4}}{\mu^{4}} .
$$

The LSP is much lighter than all other nautralinos:

$$
m_{\chi_{2}^{0}}-m_{\chi_{1}^{0}} \approx \min \left(|\mu|,\left|M_{1}\right|\right)-\left|M_{2}\right| .
$$

The composition of the LSP is given by:

$$
\begin{aligned}
& N_{11} \approx-\frac{1}{2} \frac{M_{Z}^{2}}{\mu M_{1}} s_{2 \theta_{W}} s_{2 \beta}, \\
& N_{12} \approx 1-\frac{1}{2} \frac{M_{Z}^{2}}{\mu^{2}} c_{\theta_{W}}^{2}, \\
& N_{13} \approx-\frac{M_{Z}}{\mu} c_{\theta_{W}} s_{\beta}-\frac{M_{2} M_{Z}}{\mu^{2}} c_{\theta_{W}} c_{\beta}, \\
& N_{14} \approx+\frac{M_{Z}}{\mu} c_{\theta_{W}} c_{\beta}+\frac{M_{2} M_{Z}}{\mu^{2}} c_{\theta_{W}} s_{\beta} .
\end{aligned}
$$

Substituting the above components into eq. (A.11) one can find the following leading terms of the LSP-Higgs coupling

$$
c_{h \chi \chi} \approx \frac{g_{2}}{2} c_{\theta_{W}} M_{Z} \frac{M_{2}+\mu s_{2 \beta}}{\mu^{2}} .
$$

Taking into account more subleading terms, not given in eqs. (A.37)-(A.40), one can complete the denominator in the above equation to the form $\mu^{2}-M_{2}^{2}$. The coupling $c_{Z \chi \chi}$ is the same as in eq. (A.31) with the last term in the square bracket less important because of a bigger value of $M_{1}$. 


\section{A.4 $\left|M_{1}\right| \ll|\mu|,\left|M_{2}\right|$}

The mass splittings are big and dominated by:

$$
m_{\chi_{1}^{+}}-m_{\chi_{1}^{0}} \approx m_{\chi_{2}^{0}}-m_{\chi_{1}^{0}} \approx \min \left(|\mu|,\left|M_{2}\right|\right)-\left|M_{1}\right| .
$$

The LSP is bino-dominated with the composition is given by:

$$
\begin{aligned}
& N_{11} \approx 1-\frac{1}{2} \frac{M_{Z}^{2}}{\mu^{2}} s_{\theta_{W}}^{2}, \\
& N_{12} \approx-\frac{1}{2} \frac{M_{Z}^{2}}{\mu M_{2}} s_{2 \theta_{W}} s_{2 \beta}, \\
& N_{13} \approx+\frac{M_{Z}}{\mu} s_{\theta_{W}} s_{\beta}+\frac{M_{1} M_{Z}}{\mu^{2}} s_{\theta_{W}} c_{\beta}, \\
& N_{14} \approx-\frac{M_{Z}}{\mu} s_{\theta_{W}} c_{\beta}-\frac{M_{1} M_{Z}}{\mu^{2}} s_{\theta_{W}} s_{\beta} .
\end{aligned}
$$

The LSP-Higgs coupling can be calculated in the same way as in the previous subsection and reads

$$
c_{h \chi \chi} \approx \frac{g_{1}}{2} s_{\theta_{W}} M_{Z} \frac{M_{1}+\mu s_{2 \beta}}{\mu^{2}} .
$$

Also here the denominator can be completed to the form $\mu^{2}-M_{1}^{2}$ presented in eq. (2.3). The coupling $c_{Z \chi \chi}$ is the same as in eq. (A.30) with the last term in the square bracket less important because of a bigger value of $M_{2}$.

\section{A.5 $\left|M_{i}\right| \sim|\mu|$ with $\left|M_{j}\right| \rightarrow \infty$}

In the previous subsections of the appendix we used the approximation of small mixings. The LSP was dominated by one of the gauginos or a mixture of higgsinos (with coefficients close to $\pm \frac{1}{\sqrt{2}}$ ). Now we turn to the approximation of the higgsino-gaugino mixing close to the maximal one in which the LSP component is close to $\pm \frac{1}{\sqrt{2}}$ for one of the gauginos and $\pm \frac{1}{2}$ for the higgsinos. This corresponds to the situation when the off-diagonal entries of the neutralino mass matrix are much smaller than the diagonal ones but (much) bigger then the difference between a pair of the diagonal terms. Let us start with the case of the bino-higgsino LSP. Using the approximation $\left|M_{1}\right|+|\mu| \gg M_{Z} \sin \theta_{W} \gg|| M_{1}|-| \mu||$ one finds: ${ }^{15}$

$$
\begin{aligned}
& m_{\chi_{1}^{0}} \approx \frac{\left|M_{1}\right|+|\mu|}{2}-\frac{\sqrt{2}}{2} M_{Z} s_{\theta_{W}} \sqrt{1+\zeta_{1} s_{2 \beta}} \\
&+\frac{1}{4} \frac{M_{Z}^{2} s_{\theta_{W}}^{2}}{\left|M_{1}\right|+|\mu|}\left(1-\zeta_{1} s_{2 \beta}\right)-\frac{\sqrt{2}}{8} \frac{\left(\left|M_{1}\right|-|\mu|\right)^{2}}{M_{Z} s_{\theta_{W}} \sqrt{1+\zeta_{1} s_{2 \beta}}} \\
& m_{\chi_{2}^{0}} \approx|\mu|+\frac{1}{2} \frac{M_{Z}^{2} s_{\theta_{W}}^{2}}{\left|M_{1}\right|+|\mu|}\left(1-\zeta_{1} s_{2 \beta}\right), \\
& m_{\chi_{1}^{+}} \approx|\mu|
\end{aligned}
$$

\footnotetext{
${ }^{15}$ In addition one has to assume that $\left|M_{1}\right| \lesssim|\mu|+\sqrt{2} M_{Z} s_{\theta_{W}} \sqrt{1+\zeta_{1} s_{2 \beta}}+M_{Z}^{2} s_{\theta_{W}}^{2}\left(1-\zeta_{1} s_{2 \beta}\right) /\left[2\left(\left|M_{1}\right|+\right.\right.$ $|\mu|)]$. Otherwise the LSP is higgsino-dominated with the mass given by (A.49).
} 
where, in order to make the expressions in this subsection more compact, we introduced the notation $\zeta_{i}=\operatorname{sgn}\left(\mu M_{i}\right)$. The chargino-neutralino mass difference, $m_{\chi_{1}^{+}}-m_{\chi_{1}^{0}}$ calculated from the above formulae may be negative or positive. This sign is important because it determines the electric charge of the LSP so we include the leading corrections from the finite value of $M_{2}$. With such corrections we get

$$
\begin{aligned}
m_{\chi_{1}^{+}}-m_{\chi_{1}^{0}} \approx & \frac{|\mu|-\left|M_{1}\right|}{2}+\frac{\sqrt{2}}{2} M_{Z} s_{\theta_{W}} \sqrt{1+\zeta_{1} s_{2 \beta}}-\frac{1}{4} \frac{M_{Z}^{2} s_{\theta_{W}}^{2}}{\left|M_{1}\right|+|\mu|}\left(1-\zeta_{1} s_{2 \beta}\right) \\
& -\operatorname{sgn}\left(M_{2} \mu\right) \frac{M_{Z}^{2}}{\left|M_{2}\right|} s_{2 \beta}+\frac{1}{4} \operatorname{sgn}\left(M_{1} M_{2}\right) \frac{M_{Z}^{4} s_{2 \theta_{W}}^{2}}{\mu^{2}\left|M_{2}\right|} s_{2 \beta}^{2}
\end{aligned}
$$

The composition of the lightest neutralino reads:

$$
\begin{aligned}
& N_{11} \approx \operatorname{sgn}\left(M_{1}\right)\left[-\frac{\sqrt{2}}{2}+\frac{1}{8} \frac{M_{Z} s_{\theta_{W}}}{\left|M_{1}\right|+|\mu|} \frac{1-\zeta_{1} s_{2 \beta}}{\sqrt{1+\zeta_{1} s_{2 \beta}}}+\frac{1}{4} \frac{\left|M_{1}\right|-|\mu|}{M_{Z} s_{\theta_{W}}} \frac{1}{\sqrt{1+\zeta_{1} s_{2 \beta}}}\right. \\
& +\frac{\sqrt{2}}{32} \frac{\left|M_{1}\right|-|\mu|}{\left|M_{1}\right|+|\mu|} \frac{1-\zeta_{1} s_{2 \beta}}{1+\zeta_{1} s_{2 \beta}}+\frac{\sqrt{2}}{32} \frac{\left(\left|M_{1}\right|-|\mu|\right)^{2}}{M_{Z}^{2} s_{\theta_{W}}^{2}} \frac{1}{1+\zeta_{1} s_{2 \beta}} \\
& \left.+\frac{\sqrt{2}}{128} \frac{M_{Z}^{2} s_{\theta_{W}}^{2}}{\left(\left|M_{1}\right|+|\mu|\right)^{2}}\left(1-\zeta_{1} s_{2 \beta}\right)\left(5+\frac{6}{1+\zeta_{1} s_{2 \beta}}\right)\right] \text {, } \\
& N_{13} \approx \operatorname{sgn}\left(M_{1} \mu\right)\left[-\frac{1}{2}-\frac{\sqrt{2}}{16} \frac{M_{Z} s_{\theta_{W}}}{\left|M_{1}\right|+|\mu|} \frac{1-\zeta_{1} s_{2 \beta}-4 c_{2 \beta}}{\sqrt{1+\zeta_{1} s_{2 \beta}}}-\frac{\sqrt{2}}{8} \frac{\left|M_{1}\right|-|\mu|}{M_{Z} s_{\theta_{W}}} \frac{1}{\sqrt{1+\zeta_{1} s_{2 \beta}}}\right. \\
& +\frac{1}{32} \frac{\left(\left|M_{1}\right|-|\mu|\right)^{2}}{M_{Z}^{2} s_{\theta_{W}}^{2}} \frac{1}{1+\zeta_{1} s_{2 \beta}}+\frac{1}{32} \frac{\left|M_{1}\right|-|\mu|}{\left|M_{1}\right|+|\mu|} \frac{1-\zeta_{1} s_{2 \beta}-4 c_{2 \beta}}{1+\zeta_{1} s_{2 \beta}} \\
& \left.-\frac{1}{128} \frac{M_{Z}^{2} s_{\theta_{W}}^{2}}{\left(\left|M_{1}\right|+|\mu|\right)^{2}}\left(2 \frac{1-\zeta_{1} s_{2 \beta}+8 c_{2 \beta}}{1+\zeta_{1} s_{2 \beta}}-9\left(1-\zeta_{1} s_{2 \beta}\right)-40 c_{2 \beta}\right)\right] \text {, } \\
& N_{14} \approx \frac{1}{2}+\frac{\sqrt{2}}{16} \frac{M_{Z} s_{\theta_{W}}}{\left|M_{1}\right|+|\mu|} \frac{1-\zeta_{1} s_{2 \beta}+4 c_{2 \beta}}{\sqrt{1+\zeta_{1} s_{2 \beta}}}+\frac{\sqrt{2}}{8} \frac{\left|M_{1}\right|-|\mu|}{M_{Z} s_{\theta_{W}}} \frac{1}{\sqrt{1+\zeta_{1} s_{2 \beta}}} \\
& -\frac{1}{32} \frac{\left(\left|M_{1}\right|-|\mu|\right)^{2}}{M_{Z}^{2} s_{\theta_{W}}^{2}} \frac{1}{1+\zeta_{1} s_{2 \beta}}-\frac{1}{32} \frac{\left|M_{1}\right|-|\mu|}{\left|M_{1}\right|+|\mu|} \frac{1-\zeta_{1} s_{2 \beta}+4 c_{2 \beta}}{1+\zeta_{1} s_{2 \beta}} \\
& +\frac{1}{128} \frac{M_{Z}^{2} s_{\theta_{W}}^{2}}{\left(\left|M_{1}\right|+|\mu|\right)^{2}}\left(2 \frac{1-\zeta_{1} s_{2 \beta}-8 c_{2 \beta}}{1+\zeta_{1} s_{2 \beta}}-9\left(1-\zeta_{1} s_{2 \beta}\right)+40 c_{2 \beta}\right) .
\end{aligned}
$$

Using the above equations for the $N_{1 i}$ one can find the LSP-Higgs coupling. Up to the first subleading terms one finds

$$
c_{h \chi \chi} \approx \frac{\sqrt{2} g_{1}}{8} \operatorname{sgn}\left(M_{1}\right)\left[\sqrt{1+\zeta_{1} s_{2 \beta}}-\frac{1}{\sqrt{2}} \frac{M_{Z} s_{\theta_{W}}}{\left|M_{1}\right|+|\mu|}\left(1-\zeta_{1} s_{2 \beta}\right)\right] .
$$

The formulae for the LSP composition, (A.52)-(A.54), can not be used for values of $\tan \beta$ close to 1 and opposite sign $M_{1}$ and $\mu$ because the term $\left(1+\zeta_{1} s_{2 \beta}\right) \equiv(1+$ $\left.\operatorname{sgn}\left(\mu M_{1}\right) s_{2 \beta}\right)$ present in the denominators of many terms becomes very small. The problem is caused by the fact that in such limit one of the off-diagonal terms in the neutralino 
mass matrix (after diagonalization of the higgsino sub-matrix) becomes very small and one of the assumptions discussed before eq. (A.48) is not fulfilled. However, the first subleading contributons to the $c_{h \chi \chi}$ with $\sqrt{1+\zeta_{1} s_{2 \beta}}$ in the denominator cancell out and do not contribute to (A.55).

The LSP coupling to the $Z$ boson is approximately given by

$$
c_{Z \chi \chi} \approx-\frac{g_{1}}{2 \sqrt{2}} c_{2 \beta} \frac{M_{Z}}{\left|M_{1}\right|+|\mu|} \frac{1}{\sqrt{1+\zeta_{1} s_{2 \beta}}}=\frac{g_{1}}{2 \sqrt{2}} \frac{M_{Z}}{\left|M_{1}\right|+|\mu|} \sqrt{1-\zeta_{1} s_{2 \beta}} .
$$

In the second equality we used the relation $c_{2 \beta}=-\sqrt{1+s_{2 \beta}} \sqrt{1-s_{2 \beta}}$ to show that $c_{Z \chi \chi}$ has no singularity for $\zeta_{1}=-1$ and $s_{2 \beta} \rightarrow 1$.

The case of large wino-higgsino mixing with decoupled bino is quite similar. One has to make the following changes: $M_{1} \rightarrow M_{2}, g_{1} \rightarrow g_{2}, s_{\theta_{W}} \rightarrow c_{\theta_{W}}, \zeta_{1} \rightarrow \zeta_{2}$ and to mulitply $c_{h \chi \chi}$ by -1 . Only the chargino mass is different and reads

$$
\begin{aligned}
m_{\chi_{1}^{+}} \approx & \frac{\left|M_{2}\right|+|\mu|}{2}-\frac{M_{Z} c_{\theta_{W}}}{\sqrt{2}} \sqrt{1+\zeta_{2} s_{2 \beta}} \\
& +\frac{1}{2} \frac{M_{Z}^{2} c_{\theta_{W}}^{2}}{\left|M_{2}\right|+|\mu|}\left(1-\zeta_{2} s_{2 \beta}\right)-\frac{\sqrt{2}}{8} \frac{\left(\left|M_{2}\right|-|\mu|\right)^{2}}{M_{Z} c_{\theta_{W}} \sqrt{1+\zeta_{2} s_{2 \beta}}} .
\end{aligned}
$$

The chargino-neutralino mass difference, with the leading corrections from finite $M_{1}$, is given by

$$
m_{\chi_{1}^{+}}-m_{\chi_{1}^{0}} \approx \frac{1}{4} \frac{M_{Z}^{2} c_{\theta_{W}}^{2}}{\left|M_{2}\right|+|\mu|}\left(1-\zeta_{2} s_{2 \beta}\right)+\frac{1}{4} \operatorname{sgn}\left(M_{1} M_{2}\right) \frac{M_{Z}^{4} c_{2 \theta_{W}}^{2}}{\mu^{2}\left|M_{1}\right|} s_{2 \beta}^{2} .
$$

Open Access. This article is distributed under the terms of the Creative Commons Attribution License (CC-BY 4.0), which permits any use, distribution and reproduction in any medium, provided the original author(s) and source are credited.

\section{References}

[1] P.H. Chankowski, J.R. Ellis, M. Olechowski and S. Pokorski, Haggling over the fine tuning price of LEP, Nucl. Phys. B 544 (1999) 39 [hep-ph/9808275] [INSPIRE].

[2] A. Birkedal, Z. Chacko and M.K. Gaillard, Little supersymmetry and the supersymmetric little hierarchy problem, JHEP 10 (2004) 036 [hep-ph/0404197] [INSPIRE].

[3] Z. Berezhiani, P.H. Chankowski, A. Falkowski and S. Pokorski, Double protection of the Higgs potential in a supersymmetric little Higgs model, Phys. Rev. Lett. 96 (2006) 031801 [hep-ph/0509311] [INSPIRE].

[4] A. Falkowski, S. Pokorski and M. Schmaltz, Twin SUSY, Phys. Rev. D 74 (2006) 035003 [hep-ph/0604066] [INSPIRE].

[5] S. Chang, L.J. Hall and N. Weiner, A supersymmetric twin Higgs, Phys. Rev. D 75 (2007) 035009 [hep-ph/0604076] [INSPIRE].

[6] N. Craig and K. Howe, Doubling down on naturalness with a supersymmetric twin Higgs, JHEP 03 (2014) 140 [arXiv:1312.1341] [INSPIRE]. 
[7] V. Cardoso and S. Yoshida, Superradiant instabilities of rotating black branes and strings, JHEP 07 (2005) 009 [hep-th/0502206] [INSPIRE].

[8] G. Burdman, Z. Chacko, R. Harnik, L. de Lima and C.B. Verhaaren, Colorless top partners, a $125 \mathrm{GeV}$ Higgs and the limits on naturalness, Phys. Rev. D 91 (2015) 055007 [arXiv:1411.3310] [INSPIRE].

[9] N. Arkani-Hamed and S. Dimopoulos, Supersymmetric unification without low energy supersymmetry and signatures for fine-tuning at the LHC, JHEP 06 (2005) 073 [hep-th/0405159] [INSPIRE].

[10] G.F. Giudice and A. Romanino, Split supersymmetry, Nucl. Phys. B 699 (2004) 65 [Erratum ibid. B 706 (2005) 65] [hep-ph/0406088] [INSPIRE].

[11] J.D. Wells, PeV-scale supersymmetry, Phys. Rev. D 71 (2005) 015013 [hep-ph/0411041] [INSPIRE].

[12] M.A. Ajaib, B. Dutta, T. Ghosh, I. Gogoladze and Q. Shafi, Neutralinos and sleptons at the LHC in light of muon $(g-2)_{\mu}$, arXiv: 1505.05896 [INSPIRE].

[13] M. Badziak, Z. Lalak, M. Lewicki, M. Olechowski and S. Pokorski, Upper bounds on sparticle masses from muon $g-2$ and the Higgs mass and the complementarity of future colliders, JHEP 03 (2015) 003 [arXiv: 1411.1450] [INSPIRE].

[14] G.B. Gelmini and P. Gondolo, Neutralino with the right cold dark matter abundance in (almost) any supersymmetric model, Phys. Rev. D 74 (2006) 023510 [hep-ph/0602230] [INSPIRE].

[15] N. Arkani-Hamed, A. Delgado and G.F. Giudice, The well-tempered neutralino, Nucl. Phys. B 741 (2006) 108 [hep-ph/0601041] [INSPIRE].

[16] C. Cheung, L.J. Hall, D. Pinner and J.T. Ruderman, Prospects and blind spots for neutralino dark matter, JHEP 05 (2013) 100 [arXiv:1211.4873] [INSPIRE].

[17] H. Baer, V. Barger and D. Mickelson, Direct and indirect detection of higgsino-like WIMPs: concluding the story of electroweak naturalness, Phys. Lett. B 726 (2013) 330 [arXiv:1303.3816] [INSPIRE].

[18] M. Berggren et al., Tackling light higgsinos at the ILC, Eur. Phys. J. C 73 (2013) 2660 [arXiv: 1307.3566] [INSPIRE].

[19] T. Cohen, M. Lisanti, A. Pierce and T.R. Slatyer, Wino dark matter under siege, JCAP 10 (2013) 061 [arXiv:1307.4082] [INSPIRE].

[20] T. Han, S. Padhi and S. Su, Electroweakinos in the light of the Higgs boson, Phys. Rev. D 88 (2013) 115010 [arXiv:1309.5966] [INSPIRE].

[21] P. Schwaller and J. Zurita, Compressed electroweakino spectra at the LHC, JHEP 03 (2014) 060 [arXiv: 1312.7350] [INSPIRE].

[22] H. Baer, A. Mustafayev and X. Tata, Monojets and mono-photons from light higgsino pair production at LHC14, Phys. Rev. D 89 (2014) 055007 [arXiv:1401.1162] [INSPIRE].

[23] H. Baer, A. Mustafayev and X. Tata, Monojet plus soft dilepton signal from light higgsino pair production at LHC14, Phys. Rev. D 90 (2014) 115007 [arXiv:1409.7058] [INSPIRE].

[24] C. Han, A. Kobakhidze, N. Liu, A. Saavedra, L. Wu and J.M. Yang, Probing light higgsinos in natural SUSY from monojet signals at the LHC, JHEP 02 (2014) 049 [arXiv:1310.4274] [INSPIRE]. 
[25] Z. Han, G.D. Kribs, A. Martin and A. Menon, Hunting quasidegenerate Higgsinos, Phys. Rev. D 89 (2014) 075007 [arXiv:1401.1235] [INSPIRE].

[26] M. Low and L.-T. Wang, Neutralino dark matter at 14 TeV and 100 TeV, JHEP 08 (2014) 161 [arXiv: 1404.0682] [INSPIRE].

[27] A. Anandakrishnan, L.M. Carpenter and S. Raby, Degenerate gaugino mass region and mono-boson collider signatures, Phys. Rev. D 90 (2014) 055004 [arXiv:1407.1833] [INSPIRE].

[28] N. Nagata and S. Shirai, Higgsino dark matter in high-scale supersymmetry, JHEP 01 (2015) 029 [arXiv: 1410.4549] [INSPIRE].

[29] J. Bramante, A. Delgado, F. Elahi, A. Martin and B. Ostdiek, Catching sparks from well-forged neutralinos, Phys. Rev. D 90 (2014) 095008 [arXiv:1408.6530] [InSPIRE].

[30] T.A.W. Martin and D. Morrissey, Electroweakino constraints from LHC data, JHEP 12 (2014) 168 [arXiv:1409.6322] [INSPIRE].

[31] B.S. Acharya, K. Bożek, C. Pongkitivanichkul and K. Sakurai, Prospects for observing charginos and neutralinos at a $100 \mathrm{TeV}$ proton-proton collider, JHEP 02 (2015) 181 [arXiv: 1410.1532] [INSPIRE].

[32] S. Gori, S. Jung, L.-T. Wang and J.D. Wells, Prospects for electroweakino discovery at a $100 \mathrm{TeV}$ hadron collider, JHEP 12 (2014) 108 [arXiv:1410.6287] [INSPIRE].

[33] J. Bramante et al., Relic neutralino surface at a $100 \mathrm{TeV}$ collider, Phys. Rev. D 91 (2015) 054015 [arXiv: 1412 .4789] [INSPIRE].

[34] G. Grilli di Cortona, Hunting electroweakinos at future hadron colliders and direct detection experiments, JHEP 05 (2015) 035 [arXiv:1412.5952] [INSPIRE].

[35] C. Han, D. Kim, S. Munir and M. Park, Accessing the core of naturalness, nearly degenerate higgsinos, at the LHC, JHEP 04 (2015) 132 [arXiv:1502.03734] [INSPIRE].

[36] A. Berlin, T. Lin, M. Low and L.-T. Wang, Neutralinos in vector boson fusion at high energy colliders, Phys. Rev. D 91 (2015) 115002 [arXiv:1502.05044] [INSPIRE].

[37] M. Chala, F. Kahlhoefer, M. McCullough, G. Nardini and K. Schmidt-Hoberg, Constraining dark sectors with monojets and dijets, JHEP 07 (2015) 089 [arXiv: 1503.05916] [INSPIRE].

[38] D. Barducci, A. Belyaev, A.K.M. Bharucha, W. Porod and V. Sanz, Uncovering natural supersymmetry via the interplay between the LHC and direct dark matter detection, JHEP 07 (2015) 066 [arXiv: 1504.02472] [INSPIRE].

[39] K. Blum, Y. Cui and M. Kamionkowski, An ultimate target for dark matter searches, Phys. Rev. D 92 (2015) 023528 [arXiv: 1412.3463] [INSPIRE].

[40] LUX collaboration, D.S. Akerib et al., First results from the LUX dark matter experiment at the Sanford Underground Research Facility, Phys. Rev. Lett. 112 (2014) 091303 [arXiv: 1310.8214] [INSPIRE].

[41] Planck collaboration, P.A.R. Ade et al., Planck 2015 results. XIII. Cosmological parameters, arXiv:1502.01589 [INSPIRE].

[42] U. Ellwanger, C. Hugonie and A.M. Teixeira, The next-to-minimal supersymmetric standard model, Phys. Rept. 496 (2010) 1 [arXiv:0910.1785] [INSPIRE].

[43] A.H. Chamseddine, R.L. Arnowitt and P. Nath, Locally supersymmetric grand unification, Phys. Rev. Lett. 49 (1982) 970 [INSPIRE]. 
[44] R. Barbieri, S. Ferrara and C.A. Savoy, Gauge models with spontaneously broken local supersymmetry, Phys. Lett. B 119 (1982) 343 [INSPIRE].

[45] G.F. Giudice and R. Rattazzi, Theories with gauge mediated supersymmetry breaking, Phys. Rept. 322 (1999) 419 [hep-ph/9801271] [INSPIRE].

[46] L. Randall and R. Sundrum, Out of this world supersymmetry breaking, Nucl. Phys. B 557 (1999) 79 [hep-th/9810155] [INSPIRE].

[47] G.F. Giudice, M.A. Luty, H. Murayama and R. Rattazzi, Gaugino mass without singlets, JHEP 12 (1998) 027 [hep-ph/9810442] [INSPIRE].

[48] N. Arkani-Hamed, S. Dimopoulos, G.F. Giudice and A. Romanino, Aspects of split supersymmetry, Nucl. Phys. B 709 (2005) 3 [hep-ph/0409232] [INSPIRE].

[49] A. Arvanitaki, N. Craig, S. Dimopoulos and G. Villadoro, Mini-split, JHEP 02 (2013) 126 [arXiv: 1210.0555$]$ [INSPIRE].

[50] E. Bagnaschi, G.F. Giudice, P. Slavich and A. Strumia, Higgs mass and unnatural supersymmetry, JHEP 09 (2014) 092 [arXiv: 1407.4081] [INSPIRE].

[51] M. Ibe, S. Matsumoto and T.T. Yanagida, Pure gravity mediation with $m_{3 / 2}=10-100 \mathrm{TeV}$, Phys. Rev. D 85 (2012) 095011 [arXiv: 1202.2253] [INSPIRE].

[52] L.J. Hall, Y. Nomura and S. Shirai, Spread supersymmetry with Wino LSP: gluino and dark matter signals, JHEP 01 (2013) 036 [arXiv:1210.2395] [INSPIRE].

[53] N. Arkani-Hamed, A. Gupta, D.E. Kaplan, N. Weiner and T. Zorawski, Simply unnatural supersymmetry, arXiv:1212.6971 [INSPIRE].

[54] K. Choi, A. Falkowski, H.P. Nilles and M. Olechowski, Soft supersymmetry breaking in KKLT flux compactification, Nucl. Phys. B 718 (2005) 113 [hep-th/0503216] [INSPIRE].

[55] K. Choi, K.Y. Lee, Y. Shimizu, Y.G. Kim and K.I. Okumura, Neutralino dark matter in mirage mediation, JCAP 12 (2006) 017 [hep-ph/0609132] [INSPIRE].

[56] XENON1T collaboration, E. Aprile, The XENON1T dark matter search experiment, Springer Proc. Phys. 148 (2013) 93 [arXiv:1206.6288] [INSPIRE].

[57] D.C. Malling et al., After LUX: the LZ program, arXiv:1110.0103 [INSPIRE].

[58] P. Cushman et al., Working group report: WIMP dark matter direct detection, arXiv: 1310.8327 [INSPIRE].

[59] L. Calibbi, J.M. Lindert, T. Ota and Y. Takanishi, LHC tests of light neutralino dark matter without light sfermions, JHEP 11 (2014) 106 [arXiv:1410.5730] [INSPIRE].

[60] L. Calibbi, J.M. Lindert, T. Ota and Y. Takanishi, Cornering light neutralino dark matter at the LHC, JHEP 10 (2013) 132 [arXiv:1307.4119] [INSPIRE].

[61] B.C. Allanach, SOFTSUSY: a program for calculating supersymmetric spectra, Comput. Phys. Commun. 143 (2002) 305 [hep-ph/0104145] [INSPIRE].

[62] G. Bélanger, F. Boudjema, A. Pukhov and A. Semenov, MicrOMEGAs 2.0: a program to calculate the relic density of dark matter in a generic model, Comput. Phys. Commun. 176 (2007) 367 [hep-ph/0607059] [INSPIRE].

[63] P. Grothaus, M. Fairbairn and J. Monroe, Directional dark matter detection beyond the neutrino bound, Phys. Rev. D 90 (2014) 055018 [arXiv: 1406. 5047] [INSPIRE]. 
[64] ICECuBE collaboration, M.G. Aartsen et al., Search for dark matter annihilations in the Sun with the 79-string IceCube detector, Phys. Rev. Lett. 110 (2013) 131302 [arXiv:1212.4097] [INSPIRE].

[65] H. Baer, A. Mustafayev, E.-K. Park and X. Tata, Target dark matter detection rates in models with a well-tempered neutralino, JCAP 01 (2007) 017 [hep-ph/0611387] [INSPIRE].

[66] J.L. Feng and D. Sanford, Heart of darkness: the significance of the zeptobarn scale for neutralino direct detection, JCAP 05 (2011) 018 [arXiv:1009.3934] [INSPIRE].

[67] P. Huang and C.E.M. Wagner, Blind spots for neutralino dark matter in the MSSM with an intermediate $m_{A}$, Phys. Rev. D 90 (2014) 015018 [arXiv:1404.0392] [InSPIRE].

[68] G. Jungman, M. Kamionkowski and K. Griest, Supersymmetric dark matter, Phys. Rept. 267 (1996) 195 [hep-ph/9506380] [INSPIRE].

[69] C.H. Chen, M. Drees and J.F. Gunion, A nonstandard string/SUSY scenario and its phenomenological implications, Phys. Rev. D 55 (1997) 330 [Erratum ibid. D 60 (1999) 039901] [hep-ph/9607421] [INSPIRE].

[70] A. Djouadi, M.M. Muhlleitner and M. Spira, Decays of supersymmetric particles: the program SUSY-HIT (SUspect-SdecaY-HDECAY-InTerface), Acta Phys. Polon. B 38 (2007) 635 [hep-ph/0609292] [INSPIRE].

[71] A. Djouadi, J. Kalinowski and M. Spira, HDECAY: a program for Higgs boson decays in the standard model and its supersymmetric extension, Comput. Phys. Commun. 108 (1998) 56 [hep-ph/9704448] [INSPIRE].

[72] M. Muhlleitner, A. Djouadi and Y. Mambrini, SDECAY: a Fortran code for the decays of the supersymmetric particles in the MSSM, Comput. Phys. Commun. 168 (2005) 46 [hep-ph/0311167] [INSPIRE].

[73] S.D. Thomas and J.D. Wells, Phenomenology of massive vectorlike doublet leptons, Phys. Rev. Lett. 81 (1998) 34 [hep-ph/9804359] [INSPIRE].

[74] J. Hisano, S. Matsumoto, M. Nagai, O. Saito and M. Senami, Non-perturbative effect on thermal relic abundance of dark matter, Phys. Lett. B 646 (2007) 34 [hep-ph/0610249] [INSPIRE].

[75] A. Hryczuk, R. Iengo and P. Ullio, Relic densities including Sommerfeld enhancements in the MSSM, JHEP 03 (2011) 069 [arXiv: 1010.2172] [INSPIRE].

[76] A. Hryczuk, The Sommerfeld enhancement for scalar particles and application to sfermion co-annihilation regions, Phys. Lett. B 699 (2011) 271 [arXiv:1102.4295] [INSPIRE].

[77] P. Gondolo, J. Edsjo, P. Ullio, L. Bergstrom, M. Schelke and E.A. Baltz, DarkSUSY: computing supersymmetric dark matter properties numerically, JCAP 07 (2004) 008 [astro-ph/0406204] [INSPIRE].

[78] M. Ibe, S. Matsumoto and R. Sato, Mass splitting between charged and neutral winos at two-loop level, Phys. Lett. B 721 (2013) 252 [arXiv: 1212.5989] [INSPIRE].

[79] S. Jung, Resolving the existence of Higgsinos in the LHC inverse problem, JHEP 06 (2014) 111 [arXiv: 1404.2691] [INSPIRE].

[80] CMS collaboration, Supersymmetry discovery potential in future LHC and HL-LHC running with the CMS detector, CMS-PAS-SUS-14-012 (2014). 
[81] ATLAS collaboration, Search for charginos nearly mass degenerate with the lightest neutralino based on a disappearing-track signature in pp collisions at $\sqrt{s}=8 \mathrm{TeV}$ with the ATLAS detector, Phys. Rev. D 88 (2013) 112006 [arXiv:1310.3675] [InSPIRE].

[82] CMS collaboration, Search for disappearing tracks in proton-proton collisions at $\sqrt{s}=8$ TeV, JHEP 01 (2015) 096 [arXiv: 1411.6006] [InSPIRE].

[83] http://collider-reach.web.cern.ch/collider-reach/.

[84] O. Buchmueller et al., Collider interplay for supersymmetry, Higgs and dark matter, Eur. Phys. J. C 75 (2015) 469 [arXiv: 1505.04702] [INSPIRE].

[85] K. Rolbiecki and K. Sakurai, Long-lived bino and wino in supersymmetry with heavy scalars and higgsinos, arXiv:1506.08799 [INSPIRE].

[86] K.J. de Vries et al., The pMSSM10 after LHC run 1, Eur. Phys. J. C 75 (2015) 422 [arXiv: 1504.03260] [INSPIRE].

[87] E.A. Bagnaschi et al., Supersymmetric dark matter after LHC run 1, arXiv:1508.01173 [INSPIRE]. 